\title{
Seismic Response of Long-Span Triple-Tower Suspension Bridge under Random Ground Motion
}

\author{
Chang-ke Jiao, ${ }^{1,2}$ Xin Dong, ${ }^{3}$ Ai-qun Li, ${ }^{2,4}$ Guang-dong Zhou, ${ }^{5}$ and Xiao-ping Wu ${ }^{1}$ \\ ${ }^{1}$ Engineering General Institute of Shanghai Construction Group, Shanghai 201114, China \\ ${ }^{2}$ School of Civil Engineering, Southeast University, Nanjing, Jiangsu 210096, China \\ ${ }^{3}$ Tongji Architectural Design (Group) Co., Ltd., Shanghai 200092, China \\ ${ }^{4}$ Beijing University of Civil Engineering and Architecture, Beijing 100044, China \\ ${ }^{5}$ College of Civil and Transportation Engineering, Hohai University, Nanjing 210098, China \\ Correspondence should be addressed to Chang-ke Jiao; jiaochk@126.com
}

Received 11 September 2016; Revised 2 December 2016; Accepted 28 December 2016; Published 14 February 2017

Academic Editor: Salvatore Caddemi

Copyright (C) 2017 Chang-ke Jiao et al. This is an open access article distributed under the Creative Commons Attribution License, which permits unrestricted use, distribution, and reproduction in any medium, provided the original work is properly cited.

\begin{abstract}
Multitower suspension bridge is of different style compared to the traditional suspension bridge with two towers, and consequently the dissimilarity of static and dynamic behaviors is distinct. As a special case of multitower suspension bridge, two long-span triple-tower suspension bridges have been constructed in China and the seismic random response of triple-tower suspension bridges is studied in this paper. A nonlinear dynamic analysis finite element model is established in ABAQUS and the Python language is utilized to facilitate the preprocess and postprocess during the finite element analysis. The procedure for random response calculation of structures based on the pseudoexcitation method is presented, with the initial equilibrium state of structure considered, which may be ignored for long-span bridges during calculating of stochastic response. The stationary seismic random responses of triple-tower suspension bridge under uniform excitation in firm, medium, and soft soil conditions and under spatially varying excitation in soft soil are investigated. The distribution of RMS of random responses of displacements and internal forces of the stiffening girder and towers is presented and discussed in detail. Results show that spatially variable ground motions should be considered in the stochastic analysis of triple-tower suspension bridge.
\end{abstract}

\section{Introduction}

Due to the advantages of suspension bridge, it has gained popularity throughout the world for spans up to approximately $2000 \mathrm{~m}$, and the traditional suspension bridges are of the style of three spans and two towers. There are numbers of long-span suspension bridges constructed around the world, such as Xihoumen Bridge (China, $578 \mathrm{~m}+1650 \mathrm{~m}+$ $578 \mathrm{~m}$ ), Runyang Bridge (China, $470 \mathrm{~m}+1490 \mathrm{~m}+470 \mathrm{~m})$, Tsing Ma Bridge (China, $455 \mathrm{~m}+1377 \mathrm{~m}+300 \mathrm{~m}$ ), Jiangyin Bridge (China, $328 m+1385 m+295 m$ ), Akashi Kaikyō Bridge (Japan, $960 m+1991 m+960$ m), Humber Suspension Bridge (England, $280 \mathrm{~m}+1410 \mathrm{~m}+530 \mathrm{~m}$ ). In contrast to the traditional suspension bridge, multispan suspension bridges (MSB) are present in research, since longer main span lengths are required. Thai et al. (2013) propose an analysis method considering both geometric and material nonlinearities to predict the ultimate strength and behavior of multispan suspension bridges, using the catenary elements and stability functions [1], and an example of triple-tower suspension bridge (TSB) was studied $(1500 \mathrm{~m}+3000 \mathrm{~m}+3000 \mathrm{~m}+$ $1500 \mathrm{~m}$ ). Forsberg (2001) discussed some special technical aspects of design of multispan suspension bridge, such as the continuity of suspension bridge main cables over two or more spans [2]. Yoshida et al. (2004) investigate structural characteristics of four-span suspension bridge, focusing on properties such as bending and torsional rigidity of the girder, sag ratio, and dead load [3]. The first triple-tower suspension bridge and the longest one in the world is the Taizhou Bridge (China, $390 m+1080 m+1080 m+390 m)$, and the second one is Maanshan Bridge (China, $360 \mathrm{~m}+$ $1080 \mathrm{~m}+1080 \mathrm{~m}+360 \mathrm{~m})$, which are the first time that this type of suspension bridges with main span exceeding $1000 \mathrm{~m}$ is constructed. The two triple-tower suspension 
bridges mentioned above provide important references for the analysis, design, and construction of TSB. Ruan et al. (2008) present the consideration of style selection of the mid tower and the stiffness demands for triple-tower suspension bridge in detail [4]. Yang et al. (2008) discussed the key issues in design of Taizhou Bridge [5], and Yang et al. (2010) present the key issues in design of Maanshan Bridge [6]. Jin et al. (2015) carried out the modal analysis of Ma'anshan Bridge in different construction stages and discussed the characteristic of modal [7]. Zhang et al. (2013) analyze the full-range aerostatic stability of Maanshan Bridge [8]. In the work by Jiao et al. (2011, 2013), the seismic performance of triple-tower suspension bridge was studied with time history nonlinear dynamic analysis methods in time domain, and the influence of elastic connection, fluid viscous dampers, and combination of both inelastic connections between girder and towers on the seismic responses are presented in detail $[9,10]$. In the work by Deng et al. (2008), the linear seismic response analysis was performed by using response spectrum and time history analysis method based on SAP2000 [11]. Li et al. (2015) investigate the seismic performance of different connecting configurations between the deck and pylons, which are referred to as seismic structural systems; a 1/40scale model of TSB incorporating different seismic structural systems was tested on the shake tables at Tongji University and the test results show that the seismic responses of the threepylon two-span suspension bridge under seismic loads are highly dependent on the connecting configurations between the deck and pylons and the results are in good agreement with that of numerical results [12]. Most of the aforementioned studies dealt with the determinate seismic response of TSB with spectrum method or time history method (linear or nonlinear); however, the stochastic seismic response is one of the most important behaviors of TSB, and researches on the stochastic seismic response of TSB are quite few.

Stochastic responses of structures require extensive computations effort especially when the vibration frequencies of structures are very close to each other or the external excitations are complicated. DebChaudhury and Gasparini $(1982,1988)$ present a unique strategy for the calculation of random response of MDOF based on the superposition of multimodes by incorporating the excitation filter equations (wind load or earthquake motion) into the governing equations of structures, and the variable state form of equations and ordinary differential equations are obtained and related strategies such as Runge-Kutta method are available [13, 14]. Peng and Conte (1998) present a closed-form solution for the evolutionary correlation and PSD matrices characterizing the nonstationary response of linear elastic, both classically and nonclassically damped MDOF systems subjected to a fully nonstationary earthquake ground motion process [15]. Muscolino and Alderucci (2015) present a method to evaluate in closed form the evolutionary frequency response function of classically damped linear structural systems subjected to both separable and nonseparable nonstationary excitations, with considering simultaneously both the amplitude and frequency changes [16]. Soyluk (2004) investigates the comparison of random vibration methods for multisupport seismic excitation analysis of long-span bridges, and the results reveals that the structural responses for each random vibration analysis depend largely on the intensity and frequency contents of power spectral density functions [17]. However, the calculation methods for stochastic response of structure are much complicated and Lin et al. (1997, 2004) present a novel method for the computation of stochastic response of structures under stationary or nonstationary, uniform, or spatially varying excitations pseudoexcitation method (PEM), with less computation effort required and the cross-correlations both between normal modes and between excitations being automatically calculated, in which the pseudoexcitations are employed instead of stochastic excitation $[18,19]$. The PEM has been used widely in the random analysis of structures. A number of studies have been performed in the past related to the incorporation of PEM into the commercial finite element software to simplify the usage of PEM in computation of stochastic response of complex structures, stationary or nonstationary, such as Zhang et al. (2009, 2013) [20, 21], Jiang et al. (2010) [22], Guo et al. (2013) [23], and Jia et al. (2013) [24]; their researches are vitally important for popularizing the usage of PEM.

The stochastic responses of traditional double-tower suspension bridges have been investigated in detail by many researchers. However, studies in this field for TSB are still never found and especially investigations of the spatially varying excitations and the random vibration analysis for TSB are in great necessity. In this paper, Taizhou Bridge is taken as an example to perform the researches on the stationary stochastic seismic response of TSB under uniformly and spatially varying excitations. The tool used is a commercially available three-dimensional finite element code, ABAQUS [25], which is capable of incorporating the geometric and material nonlinearity and a 3D analytical model is developed. The procedure of random vibration analysis of structures based on the PEM is presented, with the consideration of initial equilibrium state of structure considered, which is very important for long-span flexible structures. The tangent stiffness matrix at the state of static equilibrium should be used during the calculation of stochastic response of structure; however, most researches do not mention it at all or clearly and the elastic stiffness matrix may be adopted incorrectly. The problem mentioned above was accomplished properly by performing a nonlinear static analysis step before the PEM carried out in this paper. All the results are obtained in batch with the programming language of Python based on the platform of $A B A Q U S$, and the stochastic responses of TSB are discussed in detail.

\section{Finite Element Model}

The main cables of the Taizhou Bridge, connecting Taizhou and Yanzhong in China crossing the Yangtze River, have a configuration of $390 m+1080 m+1080 m+390 m$ and the rise-span ratio of each main cable is $1 / 9$ and the distance between two parallel cables is $34.8 \mathrm{~m}$ in transverse direction of the bridge, shown as Figure 1(a). Streamlined flat steel box section is employed by the stiffening girder with a height of $3.5 \mathrm{~m}$ and width of $39.1 \mathrm{~m}$, shown as Figure $1(\mathrm{~b})$. The standard distance interval of suspender is $16 \mathrm{~m}$ and the distance of 


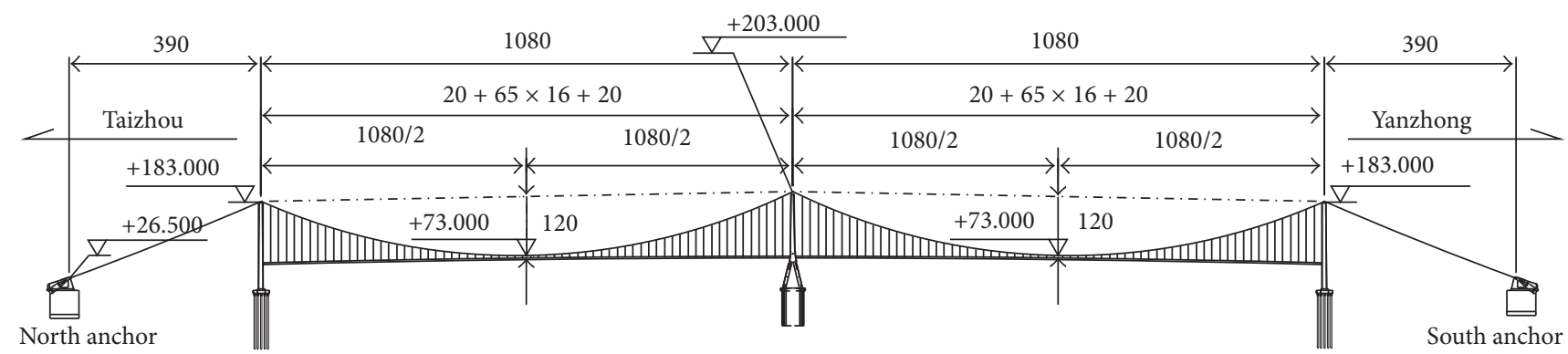

(a) Configuration of Taizhou Bridge (unit: $\mathrm{m}$ )

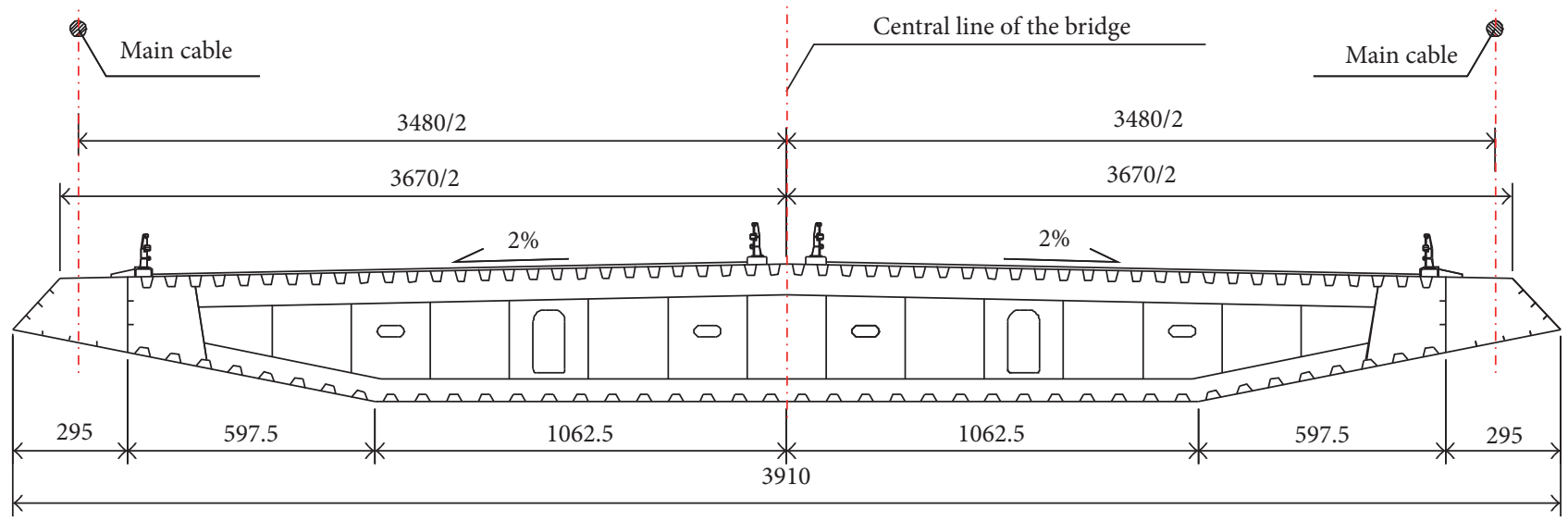

(b) Typical section of girder (unit: $\mathrm{cm}$ )

Figure 1: Schematic of Taizhou Bridge.

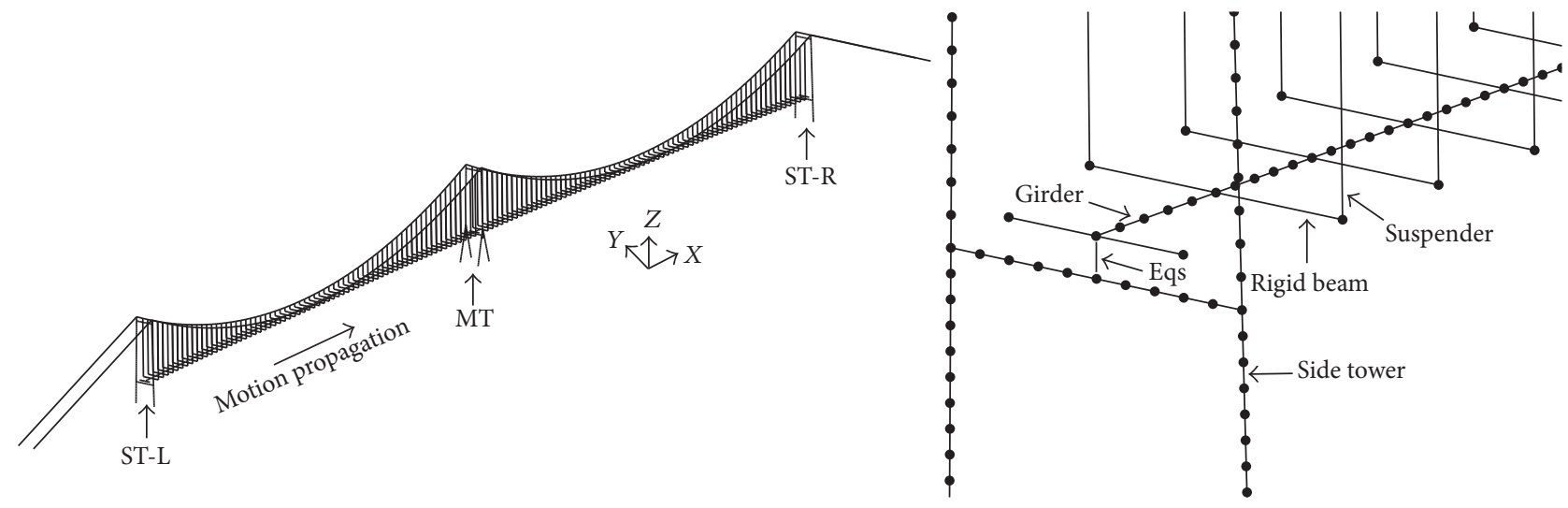

FIgURE 2: Finite element model of Taizhou Bridge.

suspender nearest to mid tower is $20 \mathrm{~m}$. The side towers are made of reinforced concrete with a height of $178 \mathrm{~m}$ and two transverse beams. The mid tower is made of steel with varying box sections and is a frame structure in transverse direction and the lower column of mid tower is herringbone in longitudinal direction.

Finite element model with beam and truss elements is established as shown in Figure 2, in which ST-L, MT, and ST$\mathrm{R}$ represent the left side tower, mid tower, and right side tower, respectively, assuming that the ground motion penetrates from ST-L to ST-R. 2-Node linear beam elements in space are employed to model the stiffening girder and towers of the bridge. 2-Node linear displacement truss elements are used for the suspenders and the main cables. These elements are readily available from $A B A Q U S$. General beam section properties are provided for the girder and tower sections and the option of No Compression is associated with the truss elements for cables and suspenders. The Ernst modules are employed for the main cables to account for the slag of cables. The main cables have a section area of $0.33 \mathrm{~m}^{2}$ and are divided by the suspenders. The stiffening girder between adjacent suspenders is divided into five beam elements. Each suspender is 


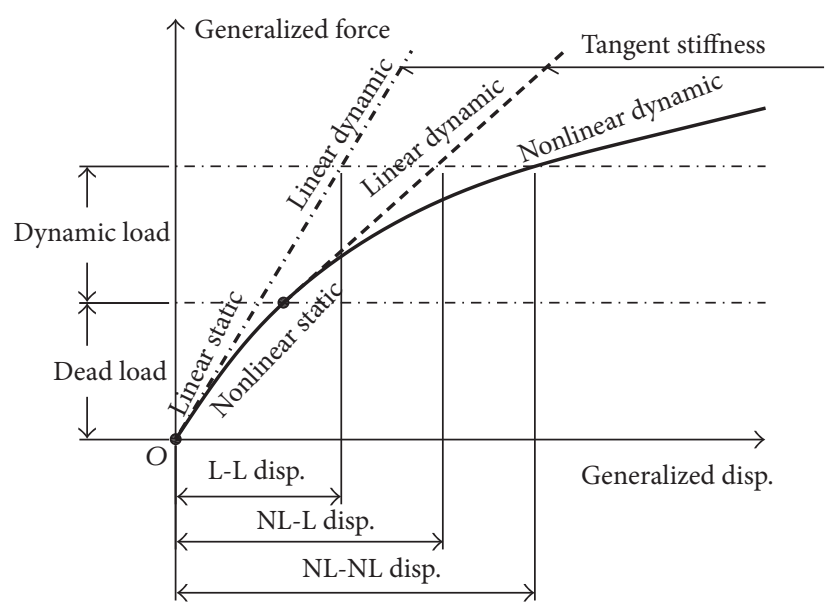

FIgURE 3: Three types of models of analysis.

modeled with one truss element. Rigid beams are employed to connect the girder and suspenders in the transverse direction. The elastic connection between mid tower and girder is modeled with spring element with the stiffness of $640 \mathrm{MN} /$ $\mathrm{m}$.

Truss element members (cables and suspenders) are hinged to beam elements (tower and rigid links). Constraint equations are established between the transverse, vertical, and rotation degrees of freedom of girder and the side towers. Constraint equations are established only between the transverse degree of freedom of girder and the lower transverse beam of mid tower, since there are no vertical bearings at the mid tower but wind shoes. The soil-structure interaction (SSI) and the shape-finding of main cables are neglected during the analysis to reduce the complexity of analysis. All degrees of freedom (DOFs) of the bottom of the towers (three translations and three rotations DOFs) are fixed and all the translation DOFs of side cables are fixed at the anchorages.

Geometric nonlinearity is one of the most important issues for long-span suspension bridges and the external loads such as dead load and prestress have an important effect on the tangent stiffness matrix of the structure. The tangent stiffness matrix of the bridge at the time of $t^{t} \mathbf{K}_{T}$ is illustrated in (1) [27], from which the effect of external loads and prestress on the initial displacement matrix ${ }^{t} \mathbf{K}_{L}$ and the initial stress matrix ${ }^{t} \mathbf{K}_{\sigma}$ can be figured out. It is important that the tangent stiffness matrix should be employed in the dynamic analysis such as the time history analysis of structure caused by earthquake or wind, which is much significant for flexible long-span structures.

$$
{ }^{t} \mathbf{K}_{T}={ }^{t} \mathbf{K}_{0}+{ }^{t} \mathbf{K}_{L}+{ }^{t} \mathbf{K}_{\sigma},
$$

where ${ }^{t} \mathbf{K}_{0}$ is the elastic matrix, depending on the coordinate of nodes and independent of the displacement of nodes; ${ }^{t} \mathbf{K}_{L}$ is the initial displacement matrix, caused by large displacement; ${ }^{t} \mathbf{K}_{\sigma}$ is the initial stress matrix, caused by the initial stress in elements.
In nonlinear dynamic analysis, the incremental finite element equilibrium equations in implicit time integration with equilibrium iterations are listed [28]:

$$
\begin{gathered}
\mathbf{M}^{t+\Delta t} \ddot{\mathbf{U}}^{(i)}+\mathbf{C}^{t+\Delta t} \dot{\mathbf{U}}^{(i)}+{ }^{t+\Delta t} \mathbf{K}_{T} \Delta \mathbf{U}^{(i)} \\
={ }^{t+\Delta t} \mathbf{R}-{ }^{t+\Delta t} \mathbf{F}^{(i-1)}, \\
{ }^{t+\Delta t} \mathbf{U}^{(i)}={ }^{t+\Delta t} \mathbf{U}^{(i-1)}+\Delta \mathbf{U}^{(i)},
\end{gathered}
$$

where $\mathbf{M}$ and $\mathbf{C}$ are the mass and damping matrix, $t$ is the time variable, and $\Delta t$ is the time increment/interval. ${ }^{t+\Delta t} \ddot{\mathbf{U}}^{(i)}$, ${ }^{t+\Delta t} \dot{\mathbf{U}}^{(i)}$, and ${ }^{t+\Delta t} \mathbf{U}^{(i-1)}+\Delta \mathbf{U}^{(i)}$ are the approximations to the acceleration, velocities, and displacements obtained in iteration ( $i$ ), respectively. The vectors of nodal point forces ${ }^{t+\Delta t} \mathbf{F}^{(i-1)}$ and ${ }^{t+\Delta t} \mathbf{K}_{T}$ are equivalent to the element stresses and tangent stiffness matrix in the configuration corresponding to the displacements ${ }^{t+\Delta t} \mathbf{U}^{(i-1)} \cdot{ }^{t+\Delta t} \mathbf{R}$ is the vector of externally applied nodal loads at time $t+\Delta t$. Once the displacements have been computed, the velocities and acceleration can be obtained from several assumptions on velocity and displacement such the trapezoidal rule, Newmark- $\beta$ method, and Bathe method [28].

Three types of analysis were performed by Abdel-Ghaffar and Nazmy (1991): (a) linear static analysis followed by linear dynamic analysis (L-L), (b) nonlinear static analysis followed by linear dynamic analysis (NL-L), and (c) nonlinear static analysis followed by nonlinear dynamic analysis (NL-NL), which are shown in Figure 3 [29]. Therefore, different results may be obtained when different starting point of the dynamic analysis is chosen.

The initial equilibrium of base state is acquired by a geometric nonlinear static analysis with initial stress applied to the main cables and gravities to all the members of structure. The frequency extraction and dynamic time history analysis can be performed based on the initial equilibrium state. Frequency extraction is performed based on the initial equilibrium state and the first six models and frequencies are shown in Figure 4, and the first model is antisymmetric lateral 


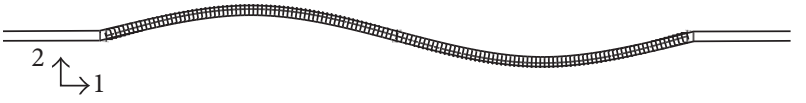

(a) $f_{1}=0.078 \mathrm{~Hz}$

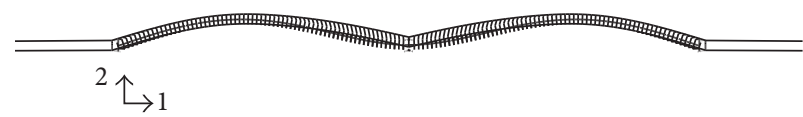

(c) $f_{3}=0.102 \mathrm{~Hz}$

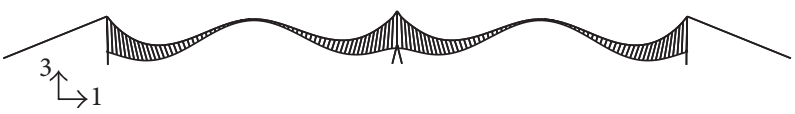

(e) $f_{5}=0.150 \mathrm{~Hz}$

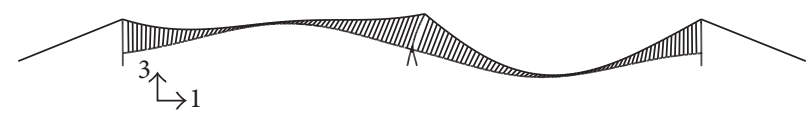

(b) $f_{2}=0.083 \mathrm{~Hz}$

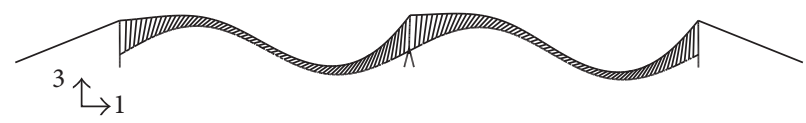

(d) $f_{4}=0.146 \mathrm{~Hz}$

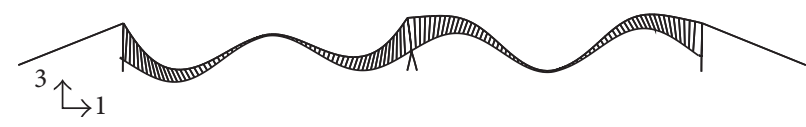

(f) $f_{6}=0.168 \mathrm{~Hz}$

FIGURE 4: The first six vibration models and frequencies of TSB.

TABLE 1: Parameters for PSD model [26].

\begin{tabular}{lccccc}
\hline Soil type & $S_{0} / \mathrm{m}^{2} \mathrm{~s}^{-3}$ & $\omega_{g} /(\mathrm{rad} / \mathrm{s})$ & $\xi_{g}$ & $\omega_{f} /(\mathrm{rad} / \mathrm{s})$ & $\xi_{f}$ \\
\hline Firm & $1.77 \times 10^{-3}$ & 15 & 0.6 & 1.5 & 0.6 \\
Medium & $2.63 \times 10^{-3}$ & 10 & 0.4 & 1 & 0.6 \\
Soft & $3.69 \times 10^{-3}$ & 5 & 0.2 & 0.5 & 0.6 \\
\hline
\end{tabular}

movement of girder and the second one is antisymmetric vertical movement of girder. The boundary conditions will be modified according to the analysis type in the proceeding step and the variables obtained are in absolute coordinate system. The assumption of Rayleigh damping is adopted in the FEM.

\section{Stochastic Model and PEM}

3.1. Model of Earthquake Excitation and Coherency. There are several power spectral density (PSD) functions for the ground acceleration such as the filtered white noise ground motion model of Kanai-Tajimi model $\left(\mathbf{S}^{\mathrm{K}-\mathrm{T}}\right)$, Clough and Penzien model ( $\left.\mathbf{S}^{\mathrm{C}-\mathrm{P}}\right)$, and Ou model [30]. The Clough and Penzien model is chosen in the paper, shown in (3a) and (3b), where $S_{0}$ is the intensity parameter, $w_{g}$ and $\xi_{g}$ are the resonant frequency and damping ratio of the first filter, and $w_{f}$ and $\xi_{f}$ are the resonant frequency and damping ratio of the second filter. Three types of soil, firm, medium, and soft, are selected and the parameters are shown in Table 1. Acceleration and displacement PSD functions for the above three types of soils are shown in Figure 5.

$$
\begin{aligned}
& S_{\ddot{u} \ddot{u}}^{\mathrm{K}-\mathrm{T}}(\omega)=\frac{\omega_{g}^{4}+4 \xi_{g}^{4} \omega_{g}^{2} \omega^{2}}{\left(\omega^{2}-\omega_{g}^{2}\right)^{2}+4 \xi_{g}^{4} \omega_{g}^{2} \omega^{2}} S_{0}, \\
& S_{\ddot{u} \ddot{u}}^{\mathrm{C}-\mathrm{P}}(\omega)=\frac{\omega^{4}}{\left(\omega^{2}-\omega_{f}^{2}\right)^{2}+4 \xi_{f}^{4} \omega_{f}^{2} \omega^{2}} S_{\ddot{u} \ddot{u}}^{\mathrm{K}-\mathrm{T}}(\omega) .
\end{aligned}
$$

In fact, the intensity parameter $S_{0}$ will decay during the propagation of ground motion. Kiureghian and Neuenhofer (1992) [31] present a model between the excitation points as
(4), where $v_{\text {app }}(\omega)$ is the apparent velocity of the earthquake motion relating to frequency $\omega$ and is usually set to a constant for simplification, since there is no suitable model and the value of $v_{\text {app }}$ may fall in a wide range according to many recent research papers. The decay of PSD is considered in the paper.

$$
S_{j k}=\sqrt{S_{j j} S_{k k}} \rho\left(d_{j k}, \omega\right) \exp \left(\frac{-i \omega d_{j k}}{v_{\mathrm{app}}(\omega)}\right) .
$$

$\mathrm{Qu}$ et al. (1996) present a model for the difference of intensity parameter $S_{0}$ between excitation points as (5), where $\Delta S_{0}$ is the increment of $S_{0}$ (unit: $\mathrm{m}^{2} \mathrm{~s}^{-3}$ ); $\Delta h$ (unit: $\mathrm{m}$ ) and $\Delta x$ (unit: $\mathrm{m}$ ) are the difference of depth and distance projected on the propagation direction between different excitation points [32]. The coherency model of QWW was present based on the average results of several different coherency models with different many ground motions records rather than on a single record. Moreover, the differences of auto-power spectral density function between spatial excitations are considered, and this model was selected during the spatial excitation analysis.

$$
\Delta S_{0}=2.571 \times 10^{-5} \Delta h-1.24 \times 10^{-6} \Delta x .
$$

There are several coherency models presented between the excitation points such as Hao model, HarichandranVanmarcke model, QWW model, Luco-Wong model, and Kiureghian model, and all of them are conditionally suitable for ground motion in general. The $Q W W$ model is chosen during the analysis in the paper. Qu et al. (1996) present a practical coherency model as (6), based on records of SMART-1, where, $a_{1}, a_{2}, b_{1}$, and $b_{2}$ are constants and $a_{1}=1.678$ $\times 10^{-5}, a_{2}=1.219 \times 10^{-3}, b_{1}=-5.5 \times 10^{-3}$, and $b_{2}=0.7674$ [32]. The coherency models of $Q W W$ are displayed in Figure 6. It is obvious that the coherencies diminish with both the distance $d_{j k}$ and frequency $\omega$.

$$
\rho\left(d_{j k}, \omega\right)=\exp \left(-\left|d_{j k}\right|^{b_{1} \omega+b_{2}}\left(a_{1} \omega^{2}+a_{2}\right)\right) .
$$



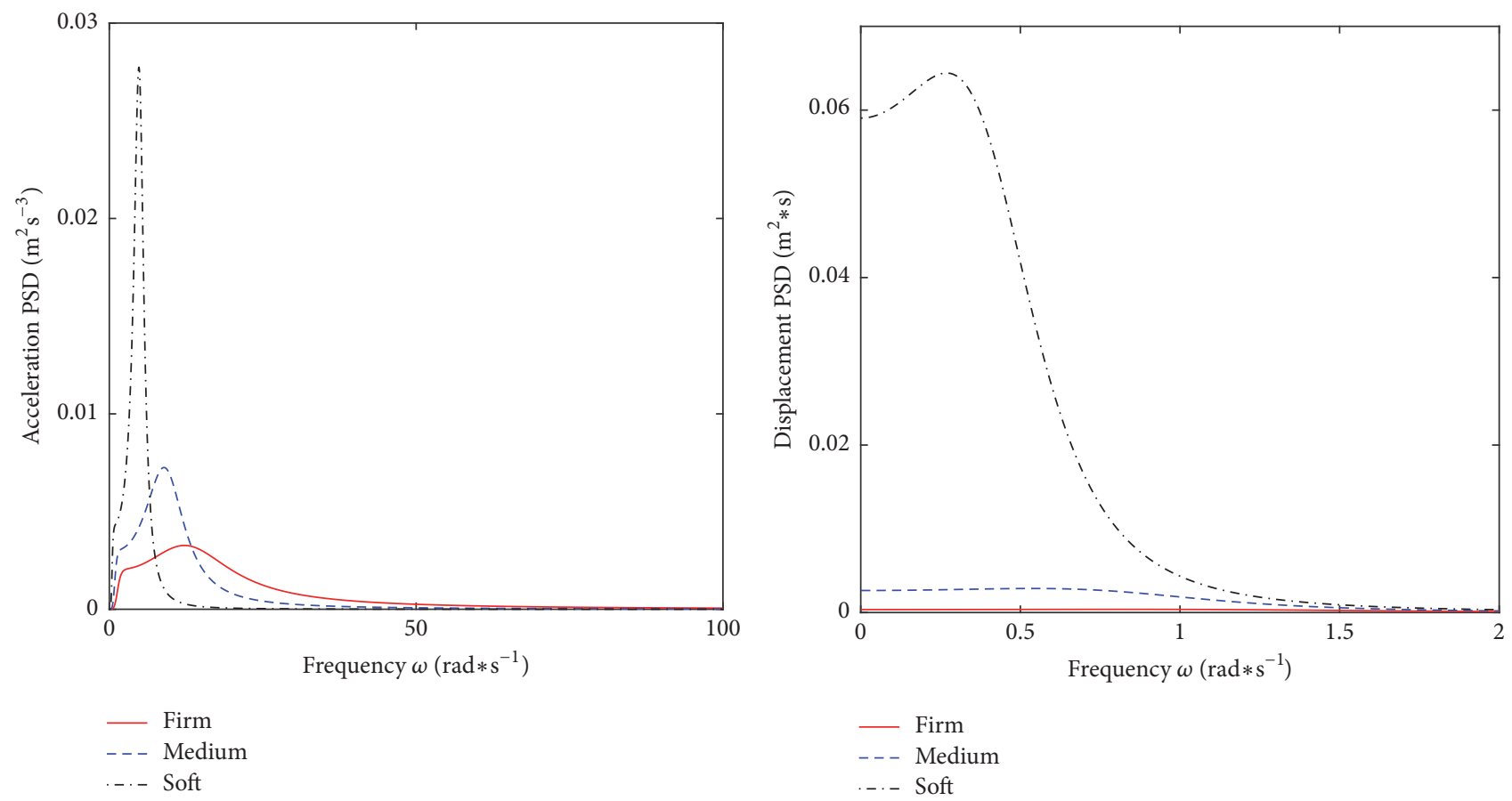

FIGURE 5: PSD of acceleration and displacement for $S^{\mathrm{C}-\mathrm{P}}$ model.
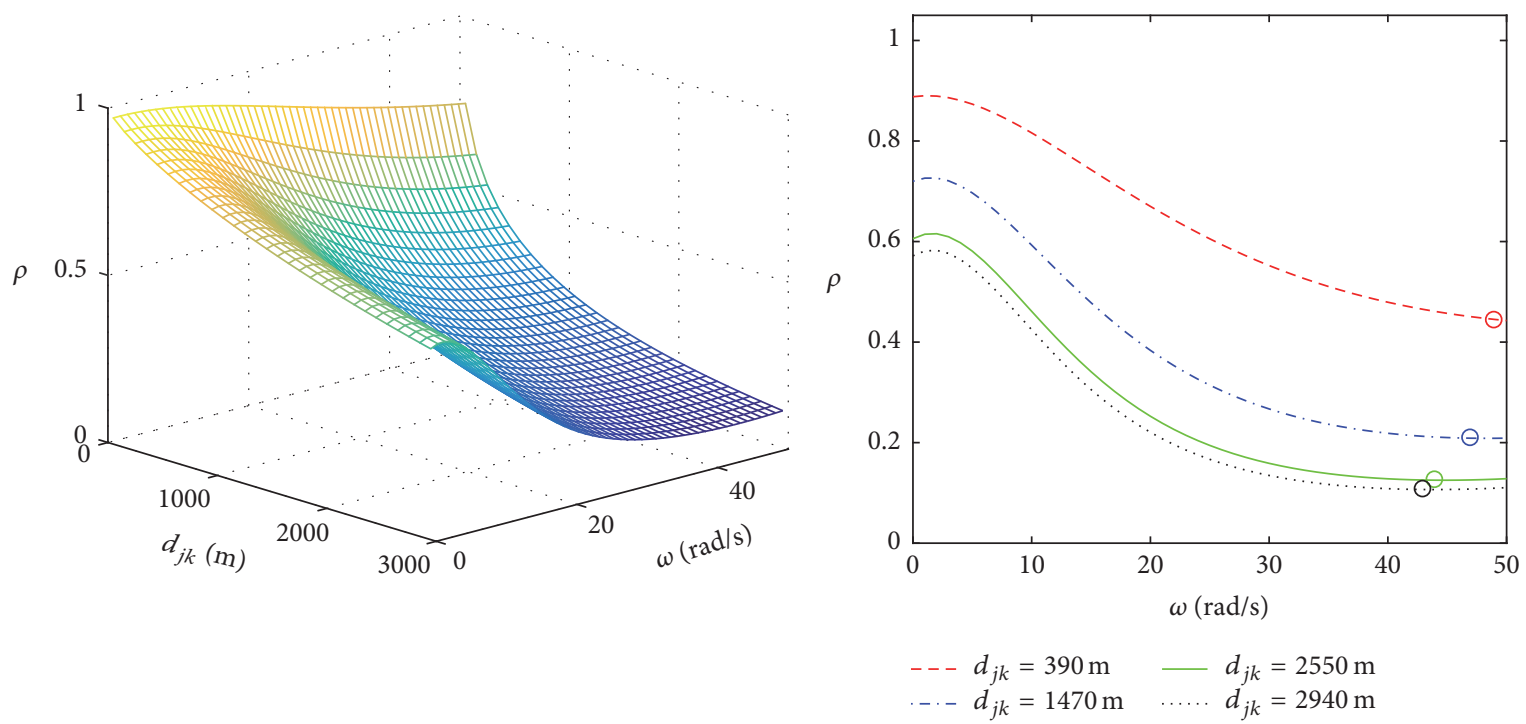

FIGURE 6: Coherency model of QWW.

3.2. Pseudoexcitation Method. The cross-PSD function matrix includes the effects of wave passage, incoherence, and site response and the elements can be expressed as

$$
\begin{array}{r}
S_{j k}(\omega)=\sqrt{S_{j j} S_{k k}}\left|\rho_{j k}\right| e^{i \omega\left(t_{j}-t_{k}\right)}, \\
\quad j, k=1,2,3, \ldots, m,
\end{array}
$$

where $S_{j j}$ is the power spectral density function of the support point $j, \rho_{j k}$ is the coherence function between support points $j$ and $k$, and $t_{j}$ is the time at which the ground motion arrives to the $j$ th excitation point. The time delay between the $j$ th and $k$ th points is $t_{j}-t_{k}=d_{j k} / v_{\text {app }}, d_{j k}$ is the projected distance between support points of $j$ and $k$ on the propagation direction, and $v_{\text {app }}$ is the apparent velocity of the earthquake motion. The item $\exp \left(i \omega\left(t_{j}-t_{k}\right)\right)$ represents the wave passage effect of ground motion.

Assume that the number of supporting points for structure is $m$ and the cross-spectral density function matrix of acceleration is given by (7b) [18-20] and the diagonal elements represent the PSD of each support point and the offline diagonal items represent the cross-spectral densities. 


$$
\mathbf{S}(\omega)=\left[\begin{array}{cccc}
S_{11} & \sqrt{S_{11} S_{22}}\left|\rho_{12}\right| e^{i \omega\left(t_{1}-t_{2}\right)} & \ldots & \sqrt{S_{11} S_{m m}}\left|\rho_{1 m}\right| e^{i \omega\left(t_{1}-t_{m}\right)} \\
\sqrt{S_{22} S_{11}}\left|\rho_{21}\right| e^{i \omega\left(t_{2}-t_{1}\right)} & S_{22} & \ldots & \sqrt{S_{22} S_{m m}}\left|\rho_{2 m}\right| e^{i \omega\left(t_{2}-t_{m}\right)} \\
\vdots & \vdots & \ddots & \vdots \\
\sqrt{S_{m m} S_{11}}\left|\rho_{m 1}\right| e^{i \omega\left(t_{m}-t_{1}\right)} & \sqrt{S_{m m} S_{22}}\left|\rho_{m 2}\right| e^{i \omega\left(t_{m}-t_{2}\right)} & \ldots & S_{m m}
\end{array}\right] .
$$

The cross-PSD function matrix is an $m$-dimensional Hermitian matrix and is usually positive or semipositive definite and can be decomposed as (7c), and the matrices J, $\mathbf{B}$, and $\mathbf{R}$ are shown in (7d), (7e), and (7f), respectively, with $\mathrm{T}$ indicating transpose and $*$ indicating complex conjugate.

$$
\begin{aligned}
& \mathbf{S}(\omega)=\left(\mathbf{B}^{*} \mathbf{J}\right) \mathbf{R}(\mathbf{J B}) \\
& \mathbf{J}=\operatorname{diag}\left[\sqrt{S_{11}}, \sqrt{S_{22}}, \ldots, \sqrt{S_{m m}}\right] \\
& =\operatorname{diag}\left[\exp \left(-i \omega t_{1}\right), \exp \left(-i \omega t_{2}\right), \ldots, \exp \left(-i \omega t_{m}\right)\right] \\
& \mathbf{R}=\left[\begin{array}{cccc}
1 & \left|\rho_{12}\right| & \ldots & \left|\rho_{1 m}\right| \\
\left|\rho_{21}\right| & 1 & \ldots & \left|\rho_{2 m}\right| \\
\vdots & \vdots & \ddots & \vdots \\
\left|\rho_{m 1}\right| & \left|\rho_{m 2}\right| & \ldots & 1
\end{array}\right] .
\end{aligned}
$$

The coherence function matrix $\mathbf{R}$ can be decomposed as $\mathbf{R}=\mathbf{Q} \mathbf{Q}^{\mathbf{T}}$, assuming that $\operatorname{rank}(\mathbf{R})=r \leq m$, and $\mathbf{Q}_{m \times r}$ can be obtained by a Cholesky decomposition. Therefore cross-PSD of excitation is $\mathbf{S}(\omega)=(\mathbf{B J Q}) *(\mathbf{B J Q})^{\mathbf{T}}$, and the pseudoexcitation can be defined as $\mathbf{P}=\mathbf{B J Q} \exp (i \omega t)$. In fact, assume that the rank of $\mathbf{S}(\omega)$ is $r(r \leq m)$, and the complex eigenvalue decomposition can be done to $\mathbf{S}(\omega)$ as (8a), where $\lambda_{k}$ and $\boldsymbol{\Phi}_{k}$ are the $k$ th eigenvalue and corresponding eigenvectors. Therefore the complex vector $\left(\lambda_{k}\right)^{0.5} \Phi_{k} \exp (i \omega t)$ can be employed as the $k$ th pseudoexcitation; all the pseudoexcitations are applied to the FEM in a Harmonic analysis step.

$$
\begin{aligned}
& \mathbf{S}(\omega) \boldsymbol{\Phi}_{k}=\lambda_{k} \boldsymbol{\Phi}_{k} \\
& \boldsymbol{\Phi}_{k}^{* \mathrm{~T}} \boldsymbol{\Phi}_{k}=\delta_{i j}= \begin{cases}1 & i=j \\
0 & i \neq j\end{cases} \\
& \mathbf{S}(\omega)=\sum_{k=1}^{r} \lambda_{k} \boldsymbol{\Phi}_{k} \boldsymbol{\Phi}_{k}^{* \mathrm{~T}} \\
& \quad=\sum_{k=1}^{r}\left[\sqrt{\lambda_{k}} \boldsymbol{\Phi}_{k}^{*} \exp (i \omega t)\right]^{*}\left[\sqrt{\lambda_{k}} \boldsymbol{\Phi}_{k}^{*} \exp (i \omega t)\right] .
\end{aligned}
$$

Assume that two arbitrary chosen variables vectors of structure are $\mathbf{x}$ and $\mathbf{y}$, which may be displacements or internal forces and so forth, and the pseudoresponses are $\widetilde{\mathbf{x}}$ and $\widetilde{\mathbf{y}}$ under the pseudoexcitations, respectively. The auto-PSD and cross-PSD can be obtained by

$$
\begin{aligned}
& \mathbf{S}_{\mathrm{xx}}=\widetilde{\mathbf{x}}^{*} \widetilde{\mathbf{x}}^{\mathrm{T}}, \\
& \mathbf{S}_{\mathrm{xy}}=\widetilde{\mathbf{x}}^{*} \widetilde{\mathbf{y}}^{\mathrm{T}}, \\
& \mathbf{S}_{\mathrm{yx}}=\widetilde{\mathbf{y}}^{*} \widetilde{\mathbf{x}}^{\mathrm{T}} .
\end{aligned}
$$

There are three parts included in calculating the stochastic response of structures using PEM. First, select the PSD and coherency model and decompose the cross-PSD matrix of excitations and compose the pseudoexcitations. Second, modify the boundary conditions of the FEM and apply the pseudoexcitations and perform serials of Harmonic analysis. Third, extract the pseudoresponse of structure and calculate PSD of variables and the variance, RMS, and so forth. Figure 7 depicts the flow chart for the procedure of stochastic response calculation with the initial equilibrium state of the structure considered [33].

The relationships between variance, autocorrelation function, and auto-power spectral density function are listed in (10a) for the random variable $X$. The basic variables focused on including the PSD, root mean square (RMS), and the cross power spectrum and covariance between variables of the seismic response of structure such as displacements, acceleration, internal forces, and the relationships are shown in (10b).

$$
\begin{aligned}
\sigma_{X}^{2}(t) & =R_{X X}(0)=E\left[X^{2}(t)\right]=\int_{-\infty}^{+\infty} S_{X}(w) d w, \\
S_{\ddot{x} \ddot{x}}(w) & =w^{4} S_{x x}(w), \\
S_{\dot{x} \ddot{x}}(w) & =i w^{3} S_{x x}(w), \\
S_{x \ddot{x}}(w) & =-w^{2} S_{x x}(w) .
\end{aligned}
$$

\section{Numerical Results}

It is assumed that the earthquake ground motions propagate from ST-L to ST-R, as illustrated in Figure 2, and the displacements and internal forces of stiffening girder and column of ST-L are discussed in detail. According to the code for seismic design of China, the pseudoexcitations are applied in three translation DOFs with the scale, 1 (longitudinal), 0.85 (transverse), and 0.65 (vertical), and the rotation DOFs of each supporting point are fixed during the Harmonic step. The notations for the outputs of the bridge are shown in 


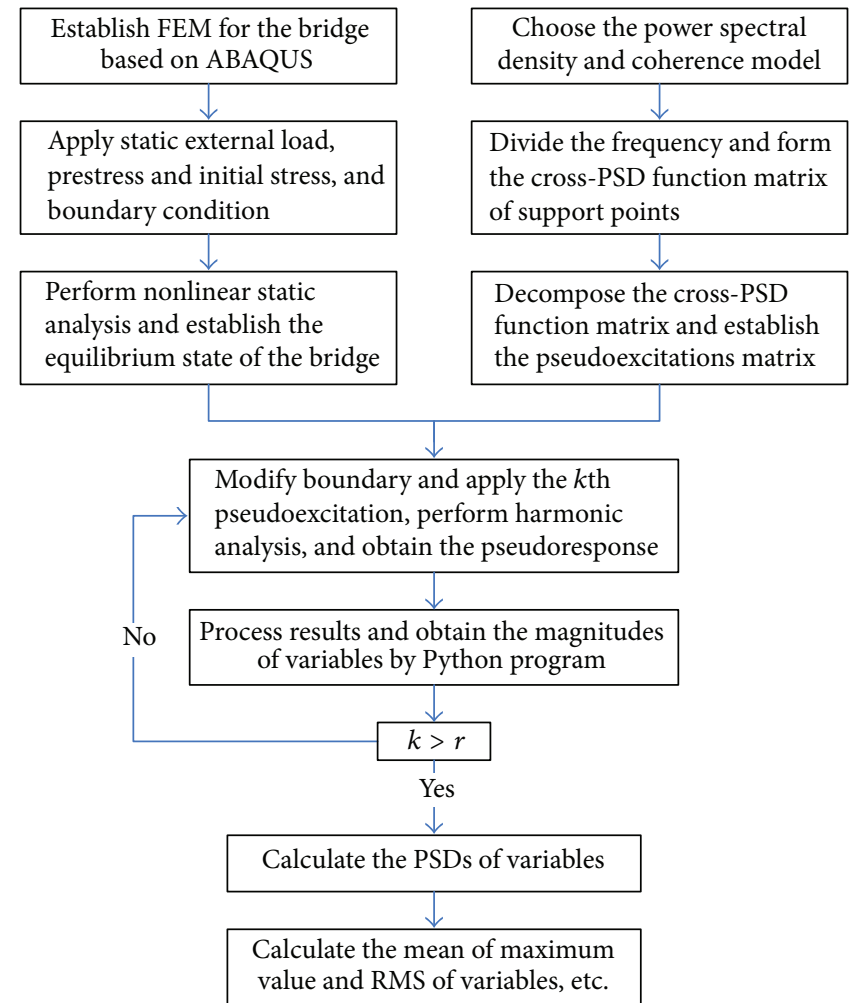

FIgURE 7: Flow chart of the procedure for PEM in ABAQUS.
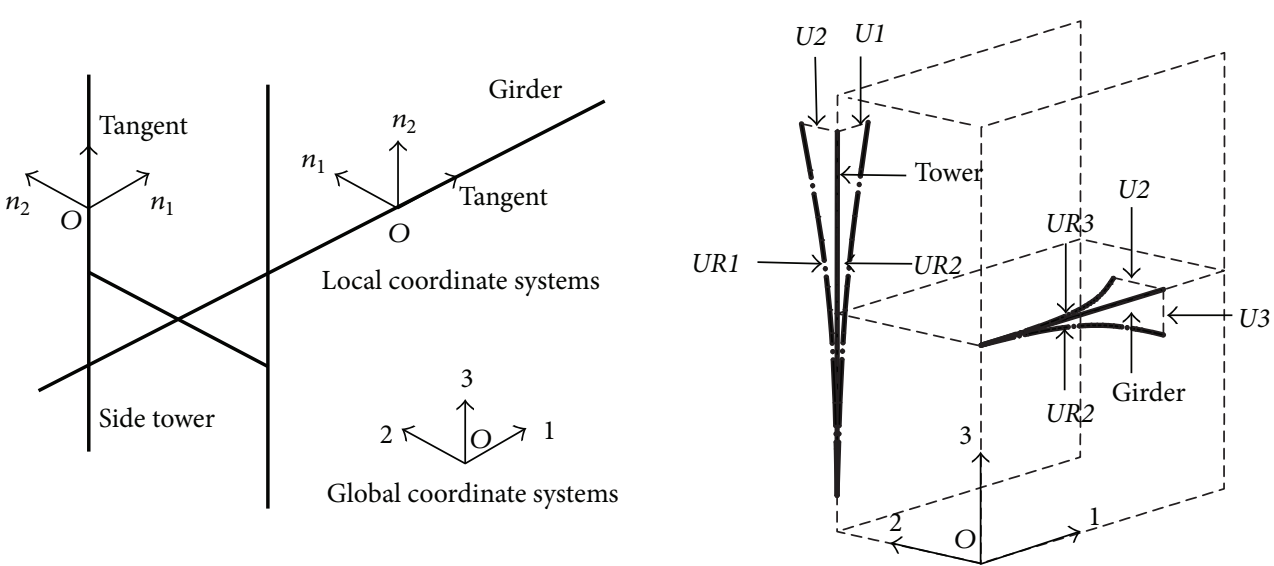

Figure 8: Notations for the displacements and internal forces of the girder and tower.

Figure 8; the displacements are in global coordinate, $O-1-2-3$, in which $U 1, U 2$, and $U 3$ are the translation displacements in longitudinal, transverse, and vertical directions of the bridge, respectively. UR1, UR2, and UR3 are the rotation displacements about the global coordinate systems axis of 1-direction, 2-direction, and 3-direction. Internal forces of element are in element local coordinate, $O-t-n_{1}-n_{2}$. SF1, SF2, and $S F 3$ are the axial force, shear force in $n_{2}$ direction, and shear force in $n_{1}$ direction, respectively. SM1 and SM2 are the element moments about $n_{1}$ axis and $n_{2}$ axis and SM3 is the element torsion about the tangent axis.
4.1. Uniform Excitation. The distribution of RMSs of internal forces and displacements of stiffening girder under uniform excitations (firm, medium, and soft soil conditions) are displayed in Figure 9, with variables notations shown in Figure 8. Results reveal the followings. (1) RMSs of variables for the condition of soft soil are larger than those corresponding to medium and firm soils, and the tendency of distributions is consistent for each variable, since the mode frequencies of the bridge are in the range of the PSD concentrating, and the PSD for soft soil is larger than that of medium and firm soils, shown in Figure 4. The RMSs of all variables along the 

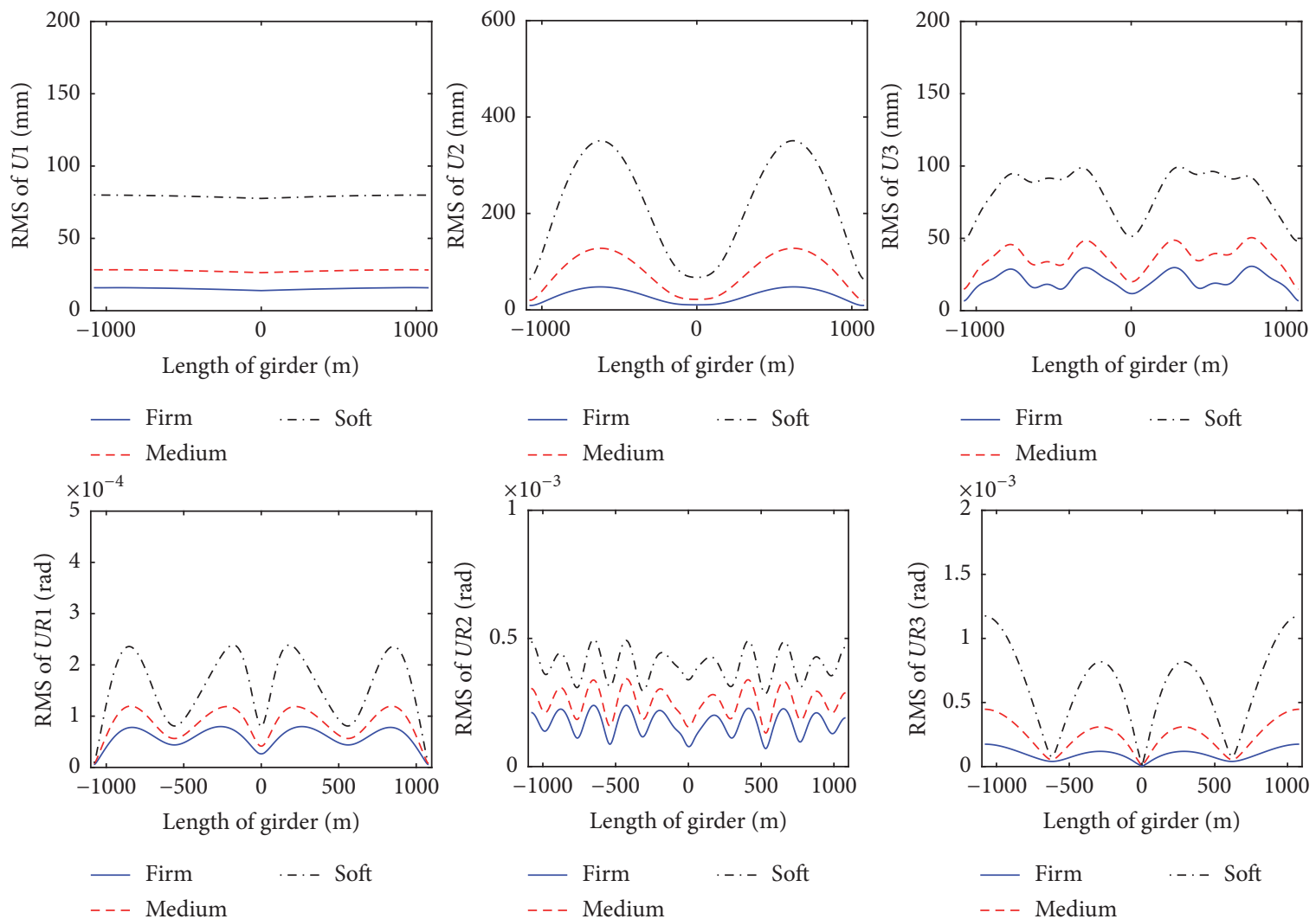

(a) RMS of displacements of girder
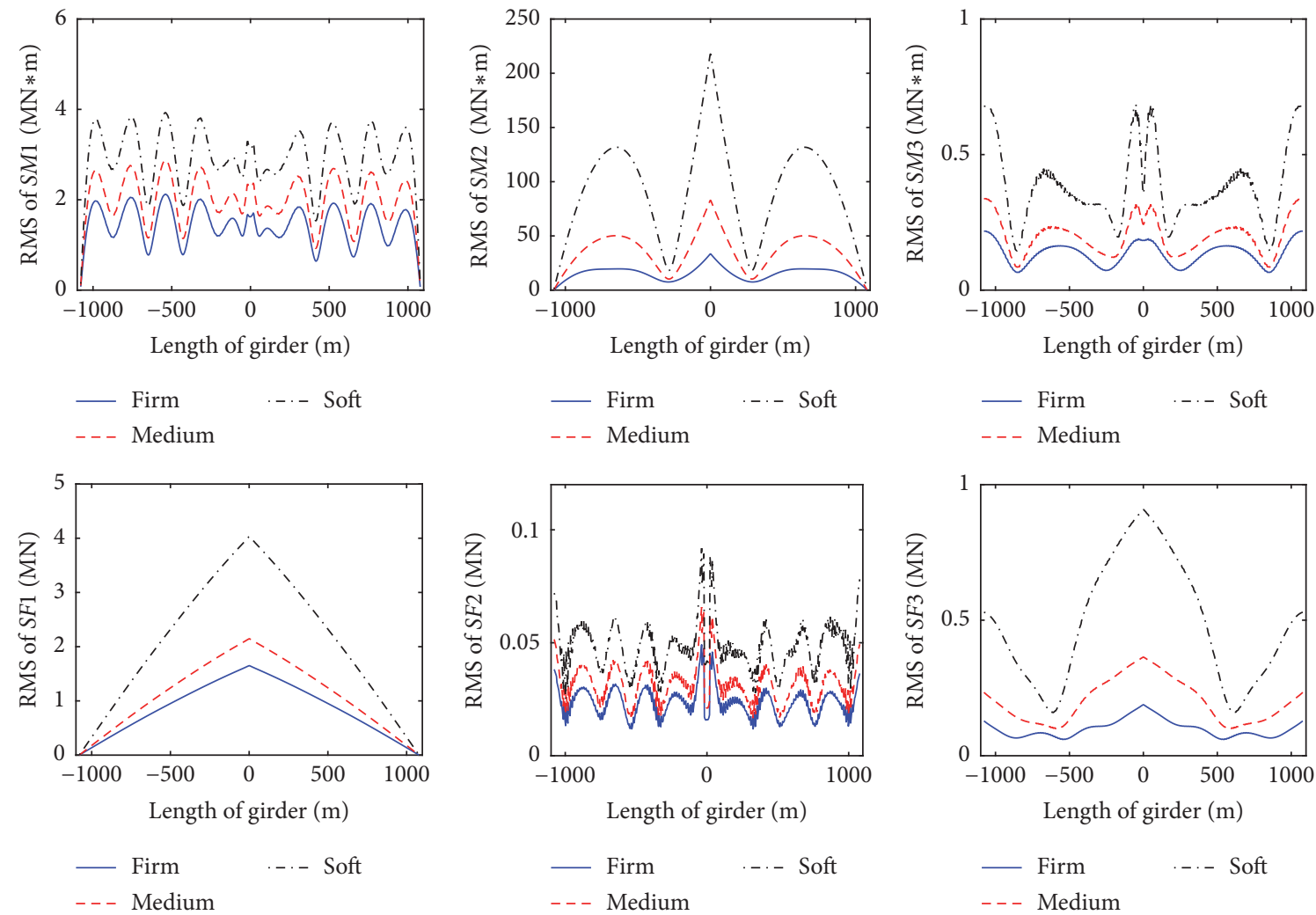

(b) RMS of internal forces of girder

FIGURE 9: RMS of random response of girder under uniform excitation. 

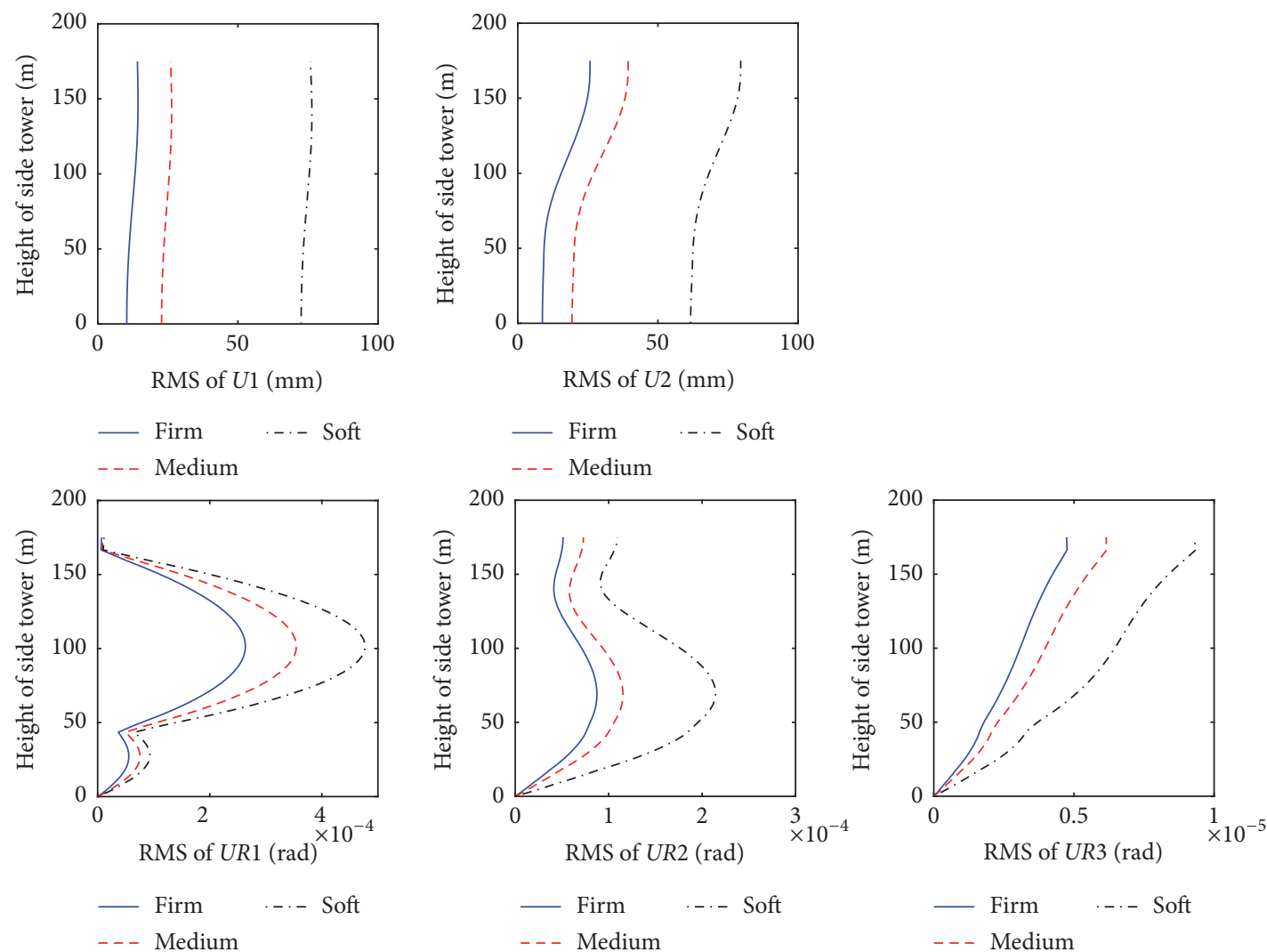

- Firm
-- Medium

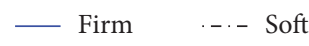

- - Medium

- Firm - - - Soft

(a) RMS of displacements of side tower
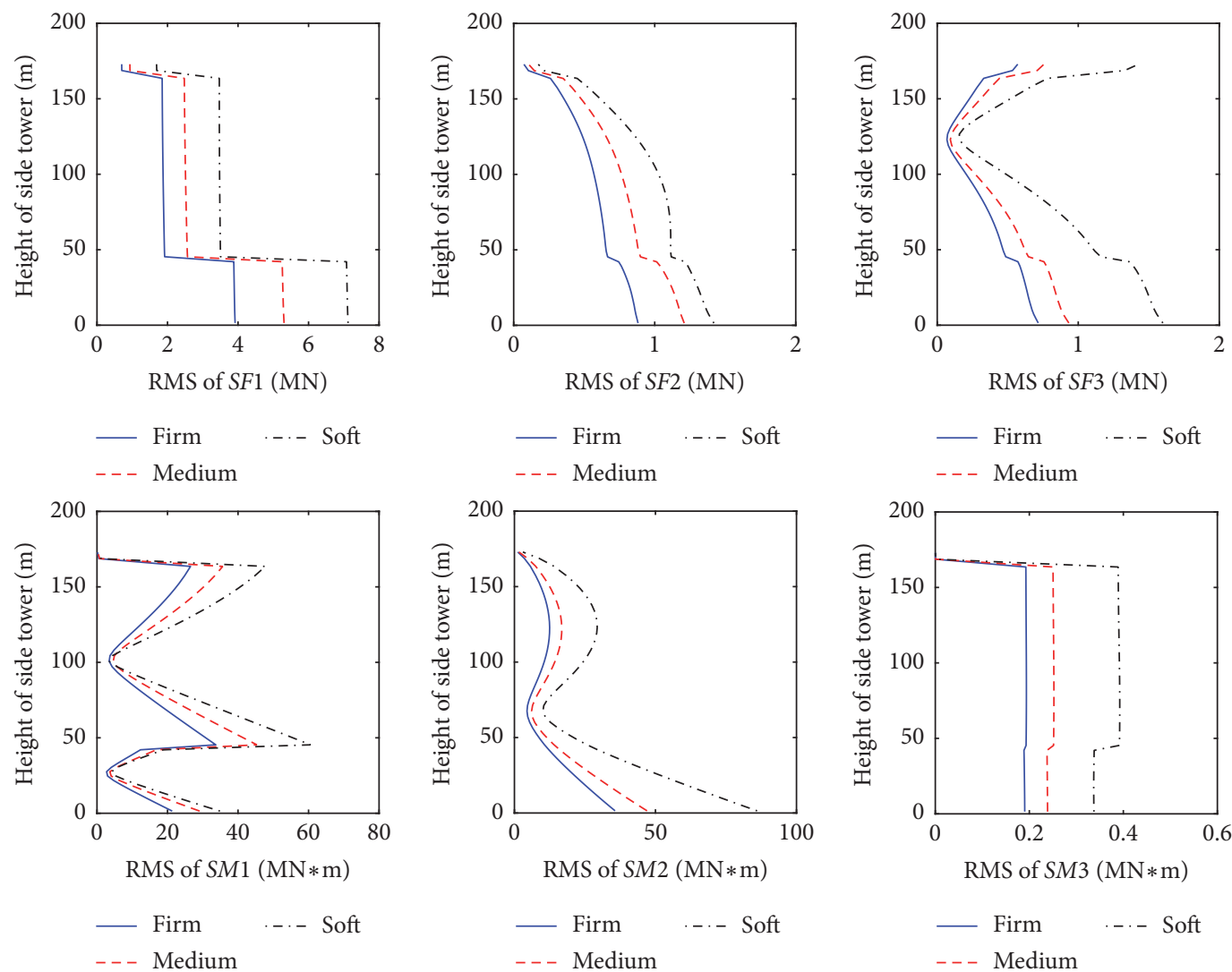

(b) RMS of internal forces of side tower

FIGURE 10: RMS of random response of side tower column under uniform excitation. 

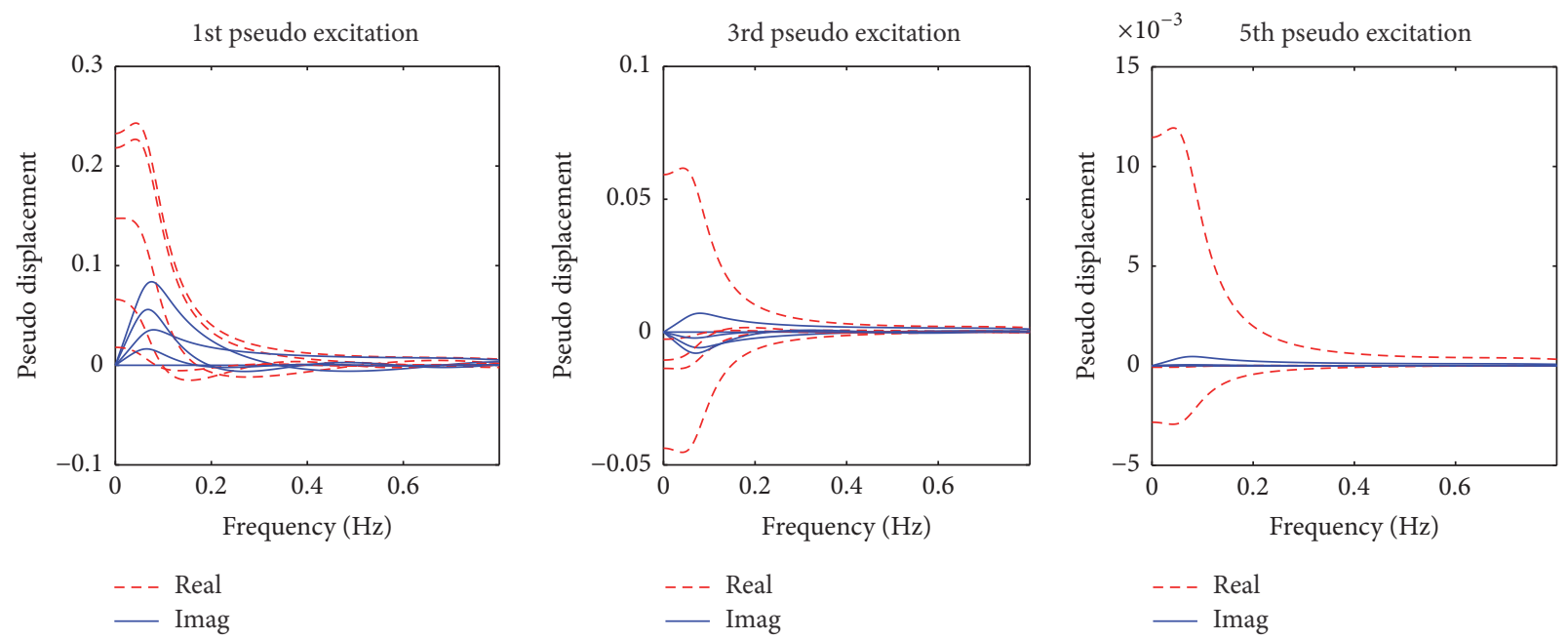

FIGURE 11: Real and image parts of pseudoexcitations.

length of girder are almost symmetrical about the mid tower. (2) Extreme values of RMS of torsional displacements, UR1, appear at the end and mid span of each span of stiffening girder and the mid tower; however, the minimum RMS of $U 2$ appears only at the end of girder and mid tower for the restrictions from towers. Notable fluctuations occur to UR2, due to the restraints from suspenders. (3) RMS of axial forces (SF1) reaches the maximum value at the mid tower because of the elastic connection between mid tower and girder, and the maximum value diminishes linearly to zero at both ends of girder. Both RMSs of shear force in vertical direction (SF2) and bending moment in vertical direction (SM1) show some fluctuations along the length of girder for the restraints of suspenders and the influence of gravity of the girder. The RMS of bending moment in transverse direction (SM2) of girder is much larger than that of SM1 or SM3 (torsion of girder) and the maximum occurs at the mid tower, which should receive much attention. There are three strong constraints on the torsional DOF of the girder at towers, and RMS of torque, SM3, of girder has peak values at the end of girder but is split into two peaks at the mid tower for the soft and medium soil conditions. (4) Three pairs of extreme RMS value of variables display alternation of peak and valley; they are (UR1,SM3), (UR2, SM1), and (UR3, SM2).

The distribution of RMSs of internal forces and displacements of side tower under uniform excitations are displayed in Figure 10. Results reveal the followings. (1) RMSs of displacements along the height of tower in longitudinal (U1) and transverse (U2) direction are small relative to the bottom of side tower because of the stiffness of pylons of tower. RMSs of $U R 1$ and $U R 2$ distribute nonlinearly along the height of side tower; however, UR3 distributes linearly along the height of side tower. (2) Abrupt changes occur to the RMS of axial force $(S F 1)$, shear in longitudinal (SF3), and shear in transverse $(S F 2)$ at the same location of side tower of lower transverse beam. RMS of SF3 reaches the minimum value at about the location of 3/4 height of side tower. (3) The RMS of SM1 varies dramatically along the height of tower, and SM2 turns out to be in R-shape along the height of tower. RMS of SM3 is much smaller than that of SM1 and SM2, since the torsional angle of the pylon is very small, and the distribution along the height of tower changes little except for the condition of soft soil.

4.2. Spatially Varying Excitation. There are five exciting points in the spatially varying excitation of Taizhou Bridge, left anchorage (ex1), left tower (ex2), mid tower (ex3), right tower (ex4), and right anchorage (ex5). Coherency model of QWW is adopted and the apparent velocity is taken as $v_{\text {app }}=$ $500 \mathrm{~m} / \mathrm{s}, 1000 \mathrm{~m} / \mathrm{s}$, and $2000 \mathrm{~m} / \mathrm{s}$, respectively. The differences of excitation points in depth are neglected; that is, $\Delta h=0$ in (5). Results of the bridge located in soil condition under spatially varying excitation are compared with those of uniform excitation. Five displacement pseudoexcitations are obtained by the method shown in Section 3.2, and the 1st, $3 \mathrm{rd}$, and 5th pseudoexcitations of real and imaginary parts are displayed in Figure 11. It is obvious that (1) the magnitude of both real and imaginary parts decays rapidly with frequency. (2) The pseudoexcitations diminish quickly along the propagation direction to the first excitation; for example, the maximum magnitude of real parts is about 0.24 at 1 st pseudoexcitation and it diminishes to about 0.012 at the 5 th pseudoexcitation.

Figure 12 illustrates a comparison of the distributions of responses of RMS, displacements, and internal forces of stiffening girder under uniformly and spatially varying excitation, with three apparent velocities. The distributions of RMS along the length of girder appear to be in the same pattern, and RMSs under spatially varying excitations are smaller than those under uniform excitations, except for the RMS of $U 3, U R 2, S F 2$, and SM1. It seems that the vertical vibration modes are sensitive to that vertical earthquake motion when subjected to spatially varying excitations. SF2 and $S M 1$ are in consistency with $U 3$ and $U R 2$, respectively, and larger vertical deformation of girder, $U 3$, will cause enlargement to $U R 2$. It seems that the smaller $v_{\text {app }}$ is, the greater the responses of $U 3, U R 2, S F 2$, and $S M 1$ are; however, the responses of other variables such as $U 2, U R 1$, and UR3 and SF3, SM2, and SM3 are on the contrary. Therefore the 

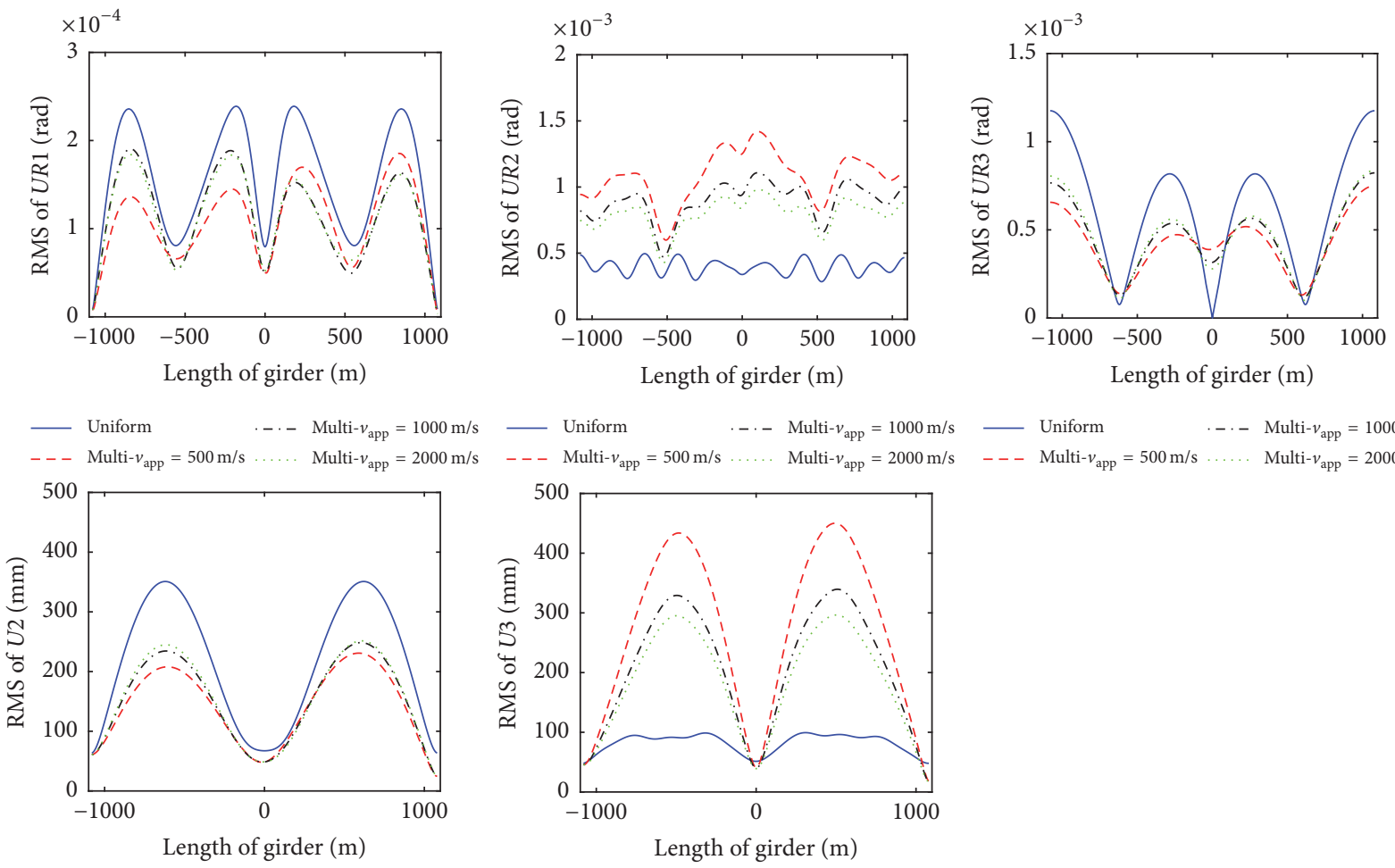

- Uniform $\quad \cdots$ Multi- $v_{\text {app }}=1000 \mathrm{~m} / \mathrm{s}$

Uniform _..- Multi- $v_{\text {app }}=1000 \mathrm{~m} / \mathrm{s}$

- - Multi- $v_{\text {app }}=500 \mathrm{~m} / \mathrm{s} \ldots \ldots$ Multi $-v_{\text {app }}=2000 \mathrm{~m} / \mathrm{s}$ - - Multi- $v_{\text {app }}=500 \mathrm{~m} / \mathrm{s} \ldots \ldots$ Multi- $v_{\text {app }}=2000 \mathrm{~m} / \mathrm{s}$

(a) RMS of displacements of girder
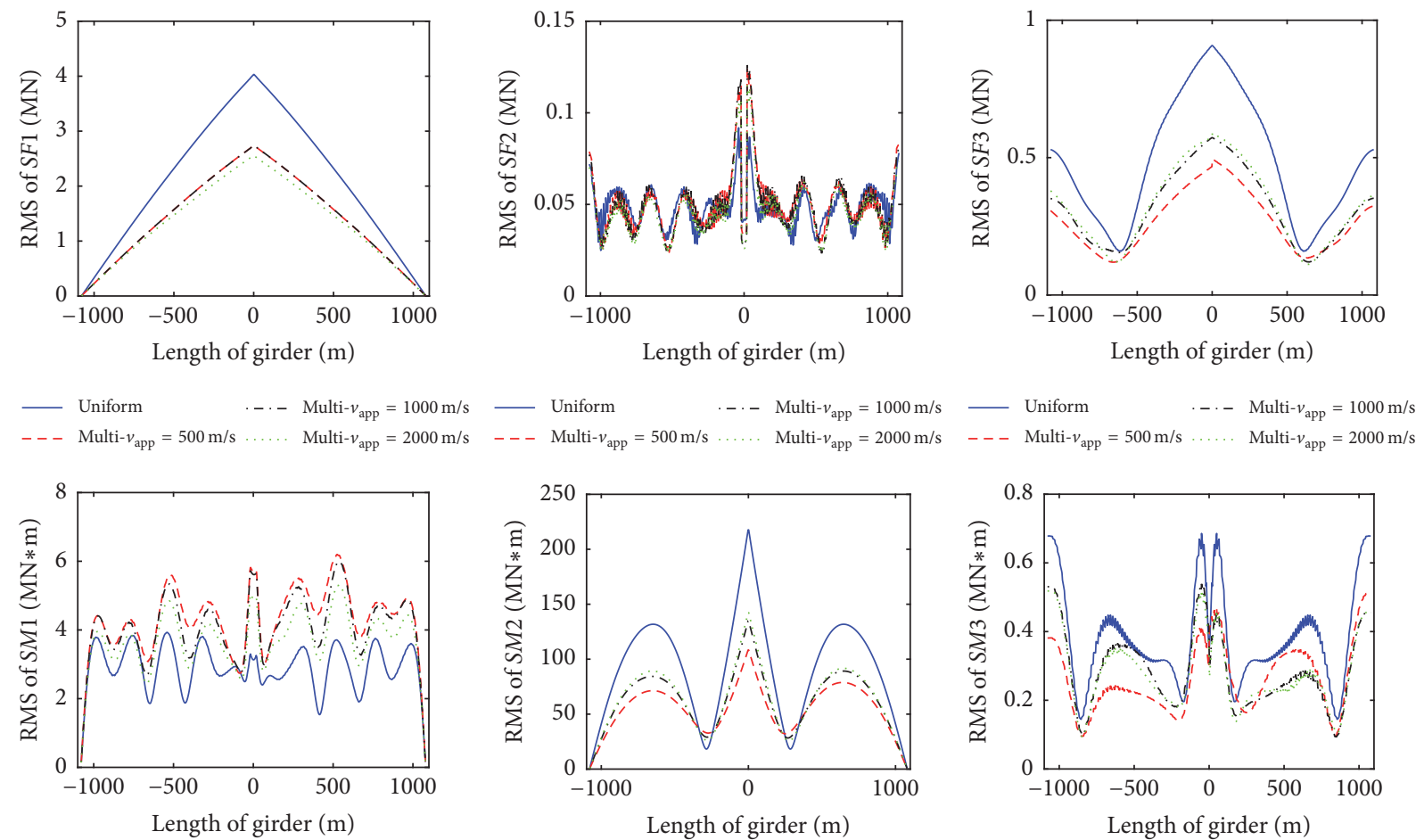

$\begin{array}{lll}- \text { Uniform } & \cdots- & \text { Multi- } v_{\text {app }}=1000 \mathrm{~m} / \mathrm{s} \\ --{\text { Multi- } v_{\text {app }}}=500 \mathrm{~m} / \mathrm{s} \ldots \ldots & \text { Multi- }-v_{\text {app }} & =2000 \mathrm{~m} / \mathrm{s}\end{array}$

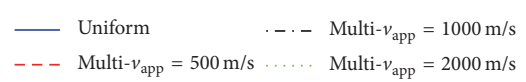

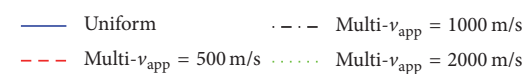

(b) RMS of internal forces of girder

FIGURE 12: Comparison of RMS of random response of girder. 

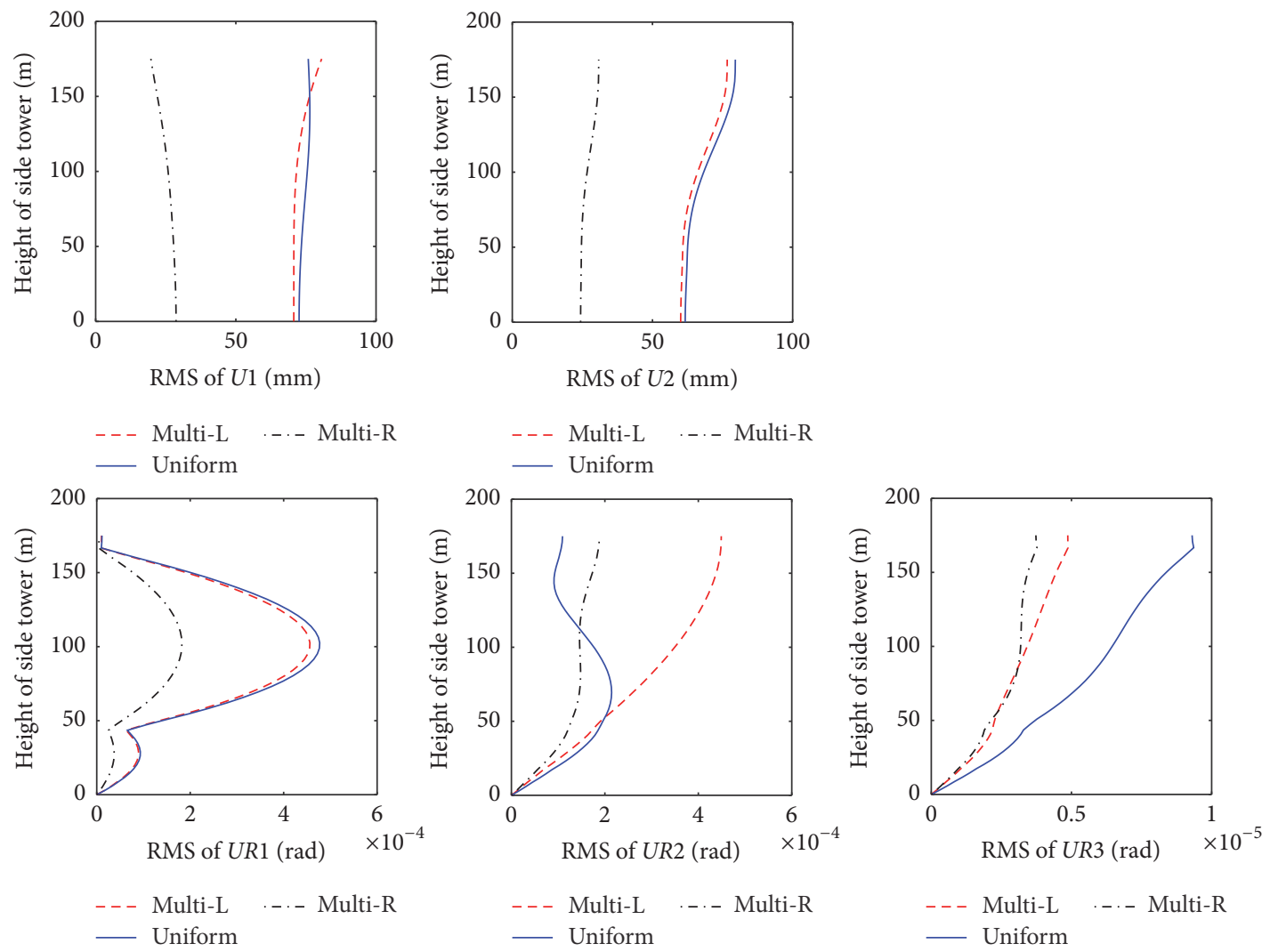

(a) RMS of displacements of side tower
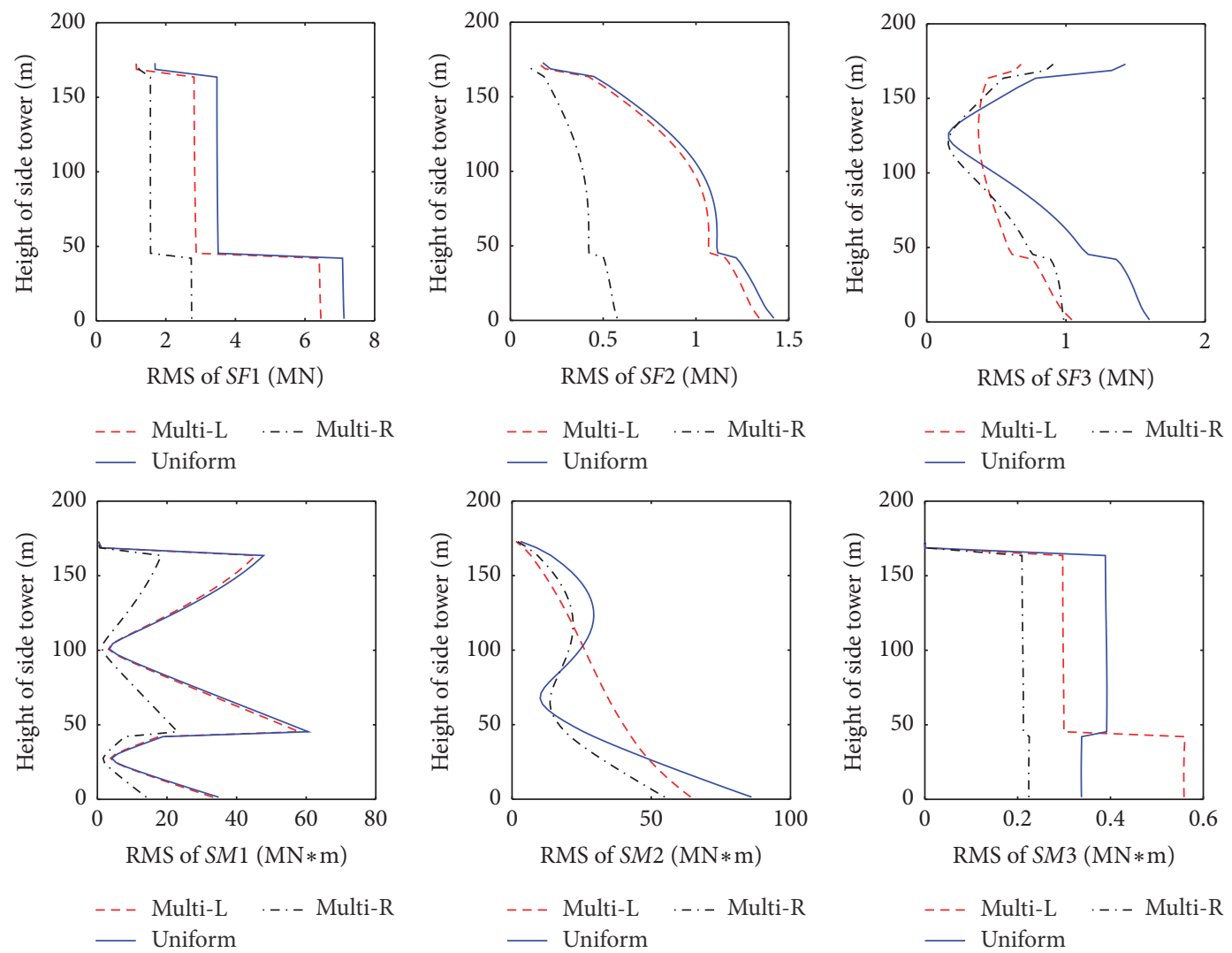

(b) RMS of internal forces of side tower

Figure 13: Comparison of RMS of random response of side tower columns. 

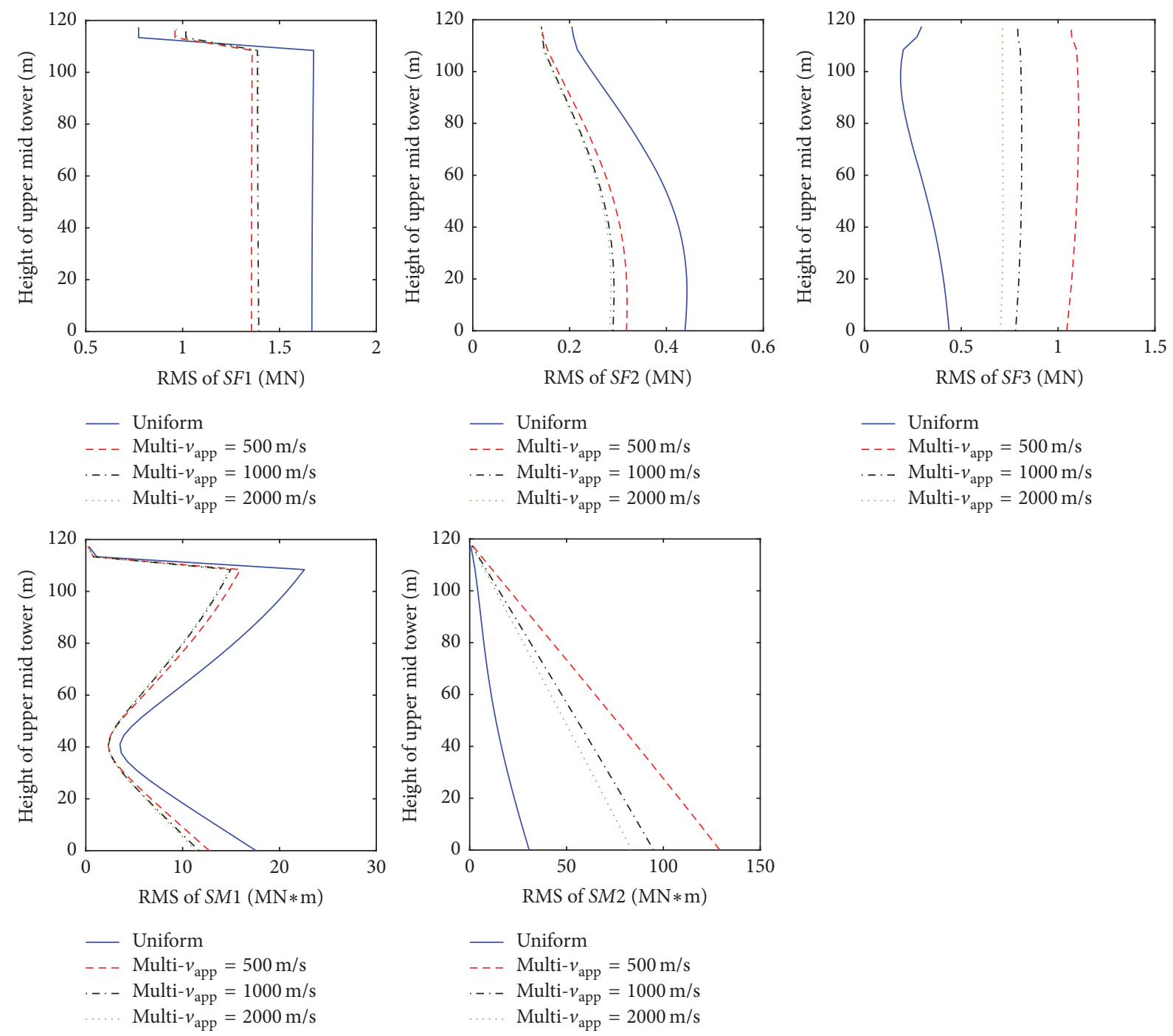

FIGURE 14: Comparison of RMS of random response of mid tower columns.

apparent velocities of the spatially varying excitations have an important effect on the stochastic responses of the bridge, which should receive much attention. The symmetry for responses of girder about the mid tower may change a little for the spatially varying excitation.

The ground motion propagates (apparent velocity is $v_{\text {app }}$ $=1000 \mathrm{~m} / \mathrm{s}$ ) form ST-L to ST-R and the comparisons of RMS of displacements and internal forces of pylons are displayed in Figure 13, in which "Multi-L" and "Multi-R" represent the responses of pylons of ST-L and ST-R, respectively. It is clear that RMSs of response of ST-L, displacements, and internal forces of pylons under spatially varying excitations are very close to those under uniformly excitation except for UR2, UR3, SF3, SM2, and SM3, since the excitation at ST-L is close to uniform excitation. RMSs of response of ST-R are much smaller than those of ST-L under spatially varying excitation for the decay of PSD of ground motion along the propagation direction. It is observed that RMSs of UR2, SF3, and SM2 of ST-R are in quiet different style from that of ST-R under spatial excitation or from that of ST-L under uniform excitation.
The comparisons of RMSs of random internal forces response of upper mid tower column are displayed in Figure 14. RMSs of $S F 1$ (axial force), SF2 (shear force in transverse direction), and SM1 (bending moment in transverse direction) of the upper mid tower column under uniform excitation are larger than those under spatially varying excitations with different apparent velocities; however, RMSs of shear force in longitudinal direction (SF3) and bending moment in longitudinal direction (SM2) under uniform excitation are smaller than those under spatially varying excitations. It seems that spatially varying excitations have more influence on mid tower in longitudinal direction compared to that in transverse direction. Results also show that the smaller apparent velocity is, the larger RMSs of bending moment in longitudinal direction (SM2) and shear force in longitudinal direction (SF3) are, and apparent velocity has smaller effects on RMSs of $S F 1, S F 2$, and SM1 than on RMSs of SF3 and SM2. It is observed that the apparent velocity of ground motion has significant influence on the stochastic responses of the bridge. 


\section{Conclusions}

The procedure for the computation of stochastic responses of long-span flexible structures under uniform and spatially varying stationary excitations based on PEM is presented, with the consideration of initial equilibrium state of structure, and is carried out by incorporating ABAQUS into Python language to calculate and process the results of random response in batch. Taizhou Bridge is taken as an example to study the character of stochastic responses of long-span multisuspension bridge such as TSB. The following conclusions can be drawn:

(1) The procedure for the computation of stochastic responses of long-span flexible structures under uniform and spatially varying stationary excitations based on PEM is presented, with the consideration of initial equilibrium state of structure.

(2) RMSs of displacements and internal forces of stiffening girder and side tower are in the same patterns when subjected to uniform excitations in the conditions of three types of soil, firm, medium, and soft. Stochastic responses of TSB are dependent on the condition of soil: the softer the soil is, the larger the stochastic responses are.

(3) The distributions of RMSs of girder and side tower are basically in the same pattern under uniform and spatially varying excitation. Example shows that RMSs of girder under spatially varying excitations are smaller than those under uniform excitations, except for the vertical displacement and related shear forces and moments in vertical direction; therefore it is difficult to conclude whether the stochastic responses of the TSB are overestimated or underestimated when the effects of spatially varying excitations are taken into account or not, since the stochastic seismic responses of TSB are dependent on the dynamic characteristic of the bridge and the PSD of excitations model, the model of coherency, and the apparent velocity. Consequently, spatially variable ground motions should be considered in the random vibration analysis of tripletower suspension bridge.

(4) RMSs of axial force, shear force in transverse direction, and bending moment in transverse direction of the upper mid tower column under uniform excitation are larger than those under spatially varying excitations with different apparent velocities and RMSs of shear force in longitudinal direction and bending moment in longitudinal direction are the opposite. Results also show that the smaller apparent velocity is, the larger RMSs of bending moment in longitudinal direction and shear force in longitudinal direction are, and apparent velocity has smaller effects on RMSs of axial force, shear force in transverse direction, and bending moment in transverse direction than on RMSs of bending moment in longitudinal direction and shear force in longitudinal direction.

\section{Competing Interests}

The authors declare that they have no competing interests.

\section{Acknowledgments}

The research for this paper was supported by (1) National Natural Science Foundation of China (Grants nos. 51408355 and 51408353) and (2) Plan of Morning Star for Young Scholars of Shanghai (Grant no. 15QB1404800).

\section{References}

[1] H. T. Thai and D. H. Choi, "Advanced analysis of multi-span suspension bridges," Journal of Constructional Steel Research, vol. 90, pp. 29-41, 2013.

[2] T. Forsberg, "Multi-span suspension bridges," Steel Structures, no. 1, pp. 63-73, 2001.

[3] O. Yoshida, M. Okuda, and T. Moriya, "Structural characteristics and applicability of four-span suspension bridge," Journal of Bridge Engineering, vol. 9, no. 5, pp. 453-463, 2004.

[4] J. Ruan, L. Ji, and P. P. Zhu, "Structure Style Selection of the Midtower of a Three-tower Suspension Bridge," Journal of Shang Dong University (Engineering Science), vol. 38, no. 2, pp. 106-111, 2008.

[5] J. Yang, G. Y. Xu, D. Z. Han, T. B. Wan, Q. F. Lu, and X. H. Luo, "Overall design and structural type selection of three-tower and two-span suspension bridge of Taizhou Changjiang River Highway Bridge," Bridge Construction, vol. 1, pp. 37-40, 2008.

[6] G. W. Yang, H. G. Xu, and Q. Zhang, "Study of key techniques for three-tower suspension bridge of Maanshan Changjiang River Bridge," Bridge Construction, vol. 5, pp. 7-11, 2010.

[7] H. Jin, X. Guo, L. Wang, and D. Feng, "Modal analysis of the triple-tower twin-span suspension bridge in deck unit erection stage," Journal of Vibroengineering, vol. 17, no. 4, pp. 2001-2012, 2015.

[8] W. M. Zhang, Y. J. Ge, and M. L. Levitan, "Nonlinear aerostatic stability analysis of new suspension bridges with multiple main spans," Journal of the Brazilian Society of Mechanical Sciences of Engineering, vol. 35, no. 2, pp. 143-151, 2013.

[9] C. K. Jiao, A. Q. Li, and H. Wang, "Seismic response control for triple-tower suspension bridges," Journal of Vibration, Measurement and Diagnosis, vol. 31, no. 2, pp. 156-161, 2011.

[10] C. K. Jiao and A. Q. Li, "Influence of inelastic connection on seismic response of triple-tower suspension bridge," China Journal of Highway and Transport, vol. 26, no. 1, pp. 98-105, 2013.

[11] Y. L. Deng, T. B. Peng, J. Z. Li, L. Ji, Z. X. Feng, and J. Ruan, "Study on dynamic characteristic and aseismic performance of a long-span triple-tower suspension bridge," Journal of Vibration and Shock, vol. 27, no. 9, pp. 105-110, 2008.

[12] J. Li, J. Yan, T. Peng, and L. Han, "Shake table studies of seismic structural systems of a taizhou changjiang highway bridge model," Journal of Bridge Engineering, vol. 20, no. 3, Article ID 04014065, 2015.

[13] A. DebChaudhury and D. A. Gasparini, "Response of MDOF systems to vector random excitation," Journal of the Engineering Mechanics Division, vol. 108, no. 2, pp. 367-385, 1982.

[14] A. DebChaudhury, "RMS response of cascaded MDOF subsystem to multiple support excitation," Journal of Engineering Mechanics, vol. 114, no. 10, pp. 1628-1650, 1988. 
[15] B. F. Peng and J. P. Conte, "Closed-form solutions for the response of linear systems to fully nonstationary earthquake excitation," Journal of Engineering Mechanics, vol. 124, no. 6, pp. 684-693, 1998.

[16] G. Muscolino and T. Alderucci, "Closed-form solutions for the evolutionary frequency response function of linear systems subjected to separable or non-separable non-stationary stochastic excitations," Probabilistic Engineering Mechanics, vol. 40, pp. 7589, 2015.

[17] K. Soyluk, "Comparison of random vibration methods for multi-support seismic excitation analysis of long-span bridges," Engineering Structures, vol. 26, no. 11, pp. 1573-1583, 2004.

[18] J. H. Lin, W. P. Shen, and F. W. Williams, "Accurate high-speed computation of non-stationary random structural response," Engineering Structures, vol. 19, no. 7, pp. 586-593, 1997.

[19] J. H. Lin, Y. H. Zhang, Q. S. Li, and F. W. Williams, "Seismic spatial effects for long-span bridges, using the pseudo excitation method," Engineering Structures, vol. 26, no. 9, pp. 1207-1216, 2004.

[20] Y. H. Zhang, Q. S. Li, J. H. Lin, and F. W. Williams, "Random vibration analysis of long-span structures subjected to spatially varying ground motions," Soil Dynamics and Earthquake Engineering, vol. 29, no. 4, pp. 620-629, 2009.

[21] C. F. Ma, Y. H. Zhang, P. Tan, D. Kennedy, F. W. Williams, and F. L. Zhou, "Non-stationary seismic response analysis of baseisolated buildings with many hysteretic devices," Computers and Structures, vol. 123, pp. 39-47, 2013.

[22] Y. Jiang, Y. Shi, and Y. Wang, "Implementation of the practical pseudo excitation method for multi-support seismic response analysis in general FEM software," Journal of Earthquake Engineering and Engineering Vibration, vol. 30, no. 1, pp. 46-52, 2010.

[23] W. Guo, Z. Yu, G. Liu, and L. Wu, "Practical solution mode of pseudo-excitation method corresponding to single and multiple point load input," Journal of Building Structures, vol. 34, no. 7, pp. 11-19, 2013.

[24] H. Y. Jia, D. Y. Zhang, S. X. Zheng, W. C. Xie, and M. D. Pandey, "Local site effects on a high-pier railway bridge under tridirectional spatial excitations: nonstationary stochastic analysis," Soil Dynamics and Earthquake Engineering, vol. 52, pp. 55-69, 2013.

[25] Hibbitt Karlson Sorensen Inc, ABAQUS/Standard User's Manual, Version 6.4.1. $t$, Hibbitt, Karlsson, and Sorensen Inc, Pawtucke, RI, USA, 2003.

[26] K. Soyluk and A. A. Dumanoglu, "Spatial variability effects of ground motions on cable-stayed bridges," Soil Dynamics and Earthquake Engineering, vol. 24, no. 3, pp. 241-250, 2004.

[27] K. J. Bathe, Finite Element Procedures, Prentice-Hall, New Jersey, NJ, USA, 1996.

[28] K. J. Bathe and M. M. Baig, "On a composite implicit time integration procedure for nonlinear dynamics," Computers \& Structures, vol. 83, no. 31-32, pp. 2513-2524, 2005.

[29] A. M. Abdel-Ghaffar and A. S. Nazmy, "3-D nonlinear seismic behavior of cable-stayed bridges," Journal of Structural Engineering, vol. 117, no. 11, pp. 3456-3476, 1991.

[30] J. P. Ou, D. T. Niu, and X. L. Du, "Random earthquake ground motion model and its parameter determination used in aseismic design," Earthquake Engineering and Engineering Vibration, vol. 11, no. 3, pp. 45-53, 1991.

[31] A. D. Kiureghian and A. Neuenhofer, "Response spectrum method for multi-support seismic excitations," Earthquake Engineering \& Structural Dynamics, vol. 21, no. 8, pp. 713-740, 1992.
[32] T. J. Qu, J. J. Wang, and Q. X. Wang, "Practical PSD model for spatially variable ground motions," Acta Seismologica Sinica, vol. 18, no. 1, pp. 55-62, 1996.

[33] C. K. Jiao and A. Q. Li, "Research on random seismic response of long-span cable-stayed bridge under multi-excitation," Journal of Vibration Engineering, vol. 26, no. 5, pp. 707-714, 2013. 


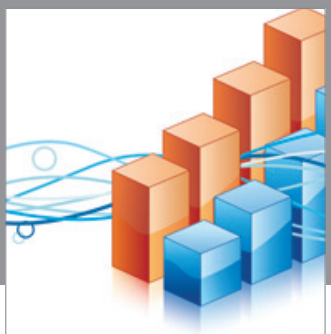

Advances in

Operations Research

vatem alat4

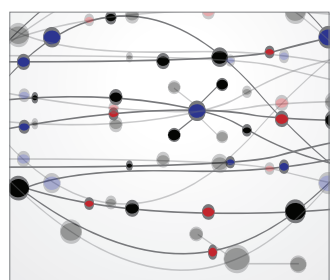

\section{The Scientific} World Journal
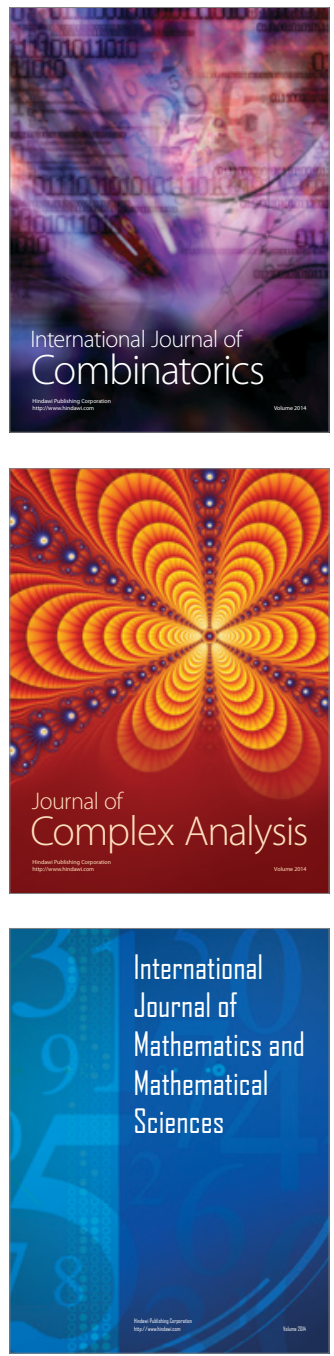
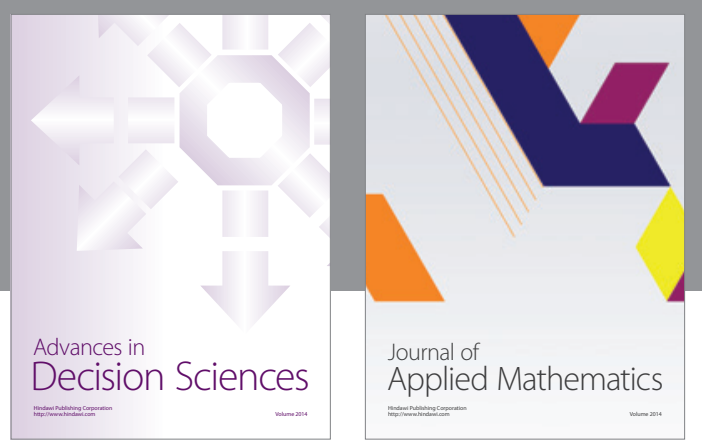

Algebra

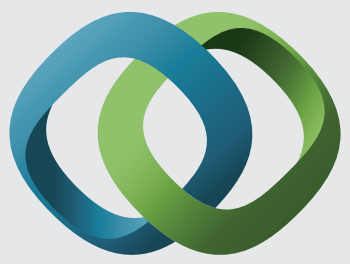

\section{Hindawi}

Submit your manuscripts at

https://www.hindawi.com
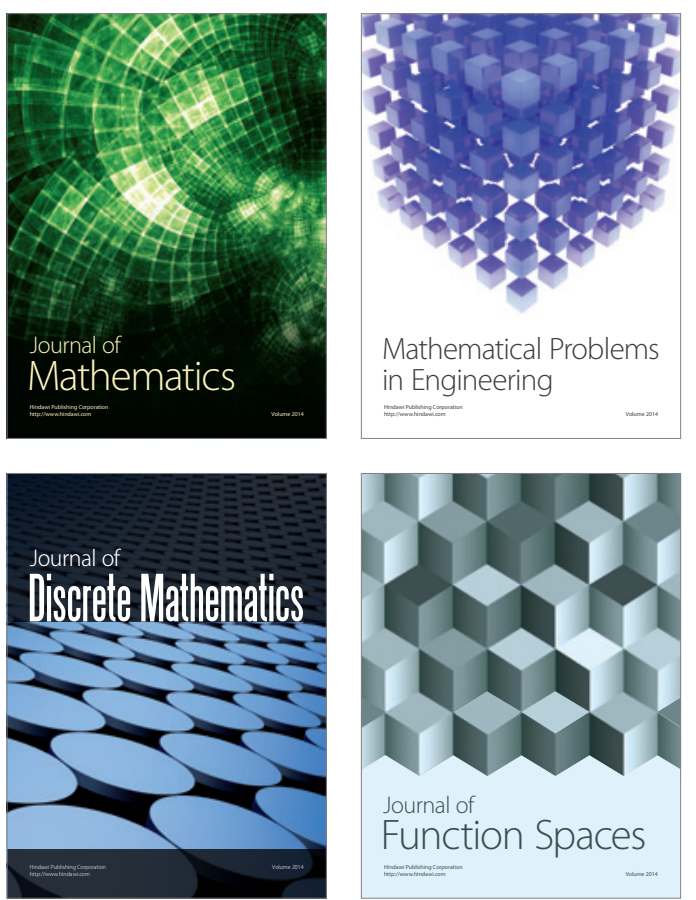

Mathematical Problems in Engineering
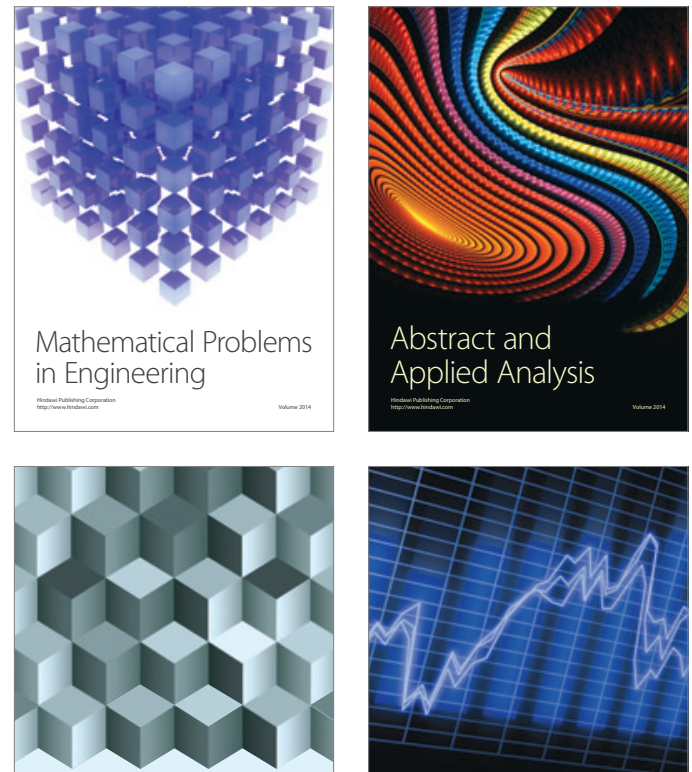

Journal of

Function Spaces

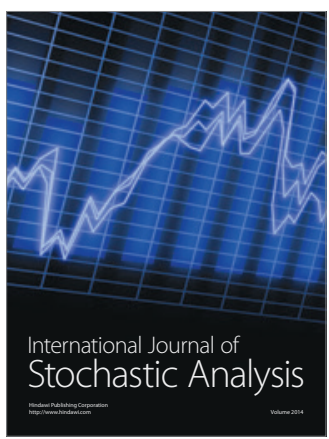

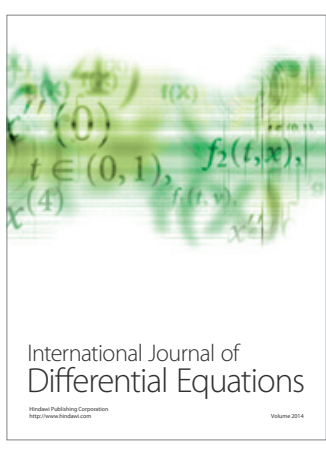
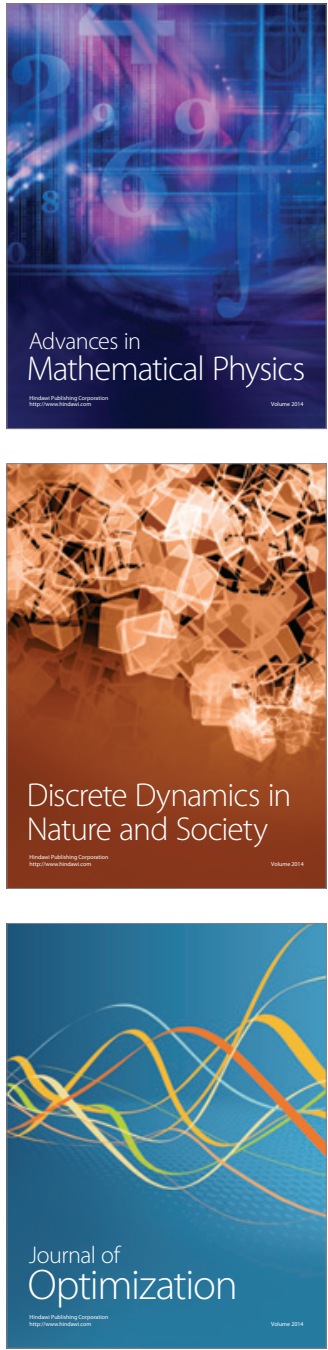